\title{
Hydrogen Bonding Catalysis Operates by Charge Stabilization in Polar Diels- Alder Reactions
}

\author{
Ruth Gordillo, ${ }^{\dagger}$ Travis Dudding, ${ }^{\ddagger}$ Christopher D. Anderson ${ }^{\dagger}$ and K. N. Houk ${ }^{*} \dagger$ \\ Department of Chemistry and Biochemistry, University of California, Los Angeles, California 90095-1569, and \\ Department of Chemistry, Brock University, St Catharines, Ontario L2S 3A1, Canada \\ houk@chem.ucla.edu
}

\section{Supporting Information}

$\begin{array}{lc}\text { A. Complete References } & S 1 \\ \text { B. Molecular Geometries and Energies } & S 2 \\ \text { C. MP2 Energies } & S 16 \\ \text { D. Charges } & S 17 \\ \text { E. Hydrogen Bond Strength Increases as Enolate Character Develops. } & S 18\end{array}$

\section{A. Complete References}

Reference 7g: Frisch, M. J.; Trucks, G. W.; Schlegel, H. B.; Scuseria, G. E.; Robb, M. A.; Cheeseman, J. R.; Zakrzewski, V. G.; Montgomery, J. A. Jr;; Stratmann, R. E.; Burant, J. C.; Dapprich, S.; Millam, J. M.; Daniels, A. D.; Kudin, K. N.; Strain, M. C.; Farkas, O.; Tomasi, J.; Barone, V.; Cossi, M.; Cammi, R.; Mennucci, B.; Pomelli, C.; Adamo, C.; Clifford, S.; Ochterski, J.; Petersson, G. A.; Ayala, P. Y.; Cui, Q.; Morokuma, K.; Malick, D. K.; Rabuck, A. D.; Raghavachari, K.; Foresman, J. B.; Cioslowski, J.; Ortiz, J. V.; Stefanov, B. B.; Liu, G.; Liashenko, A.; Piskorz, P.; Komaromi, I. R.; Gomperts, R.; Martin, L.; Fox, D. J.; Keith, T.; Al-Laham, M. A.; Peng, C. Y.; Nanayakkara, A.; Gonzalez, C.; Challacombe, M. P.; Gill, M. W.; Johnson, B.; Chen, W.; Wong, M. W.; Andres, J. L.; Gonzalez, C.; Head-Gordon, M.; Replogle, E. S.; Pople, J. A. Gaussian 98, revision A.6; Gaussian, Inc.: Pittsburgh, PA, 1998.

Reference 7h: Frisch, M. J.; Trucks, G. W.; Schlegel, H. B.; Scuseria, G. E.; Robb, M. A.; Cheeseman, J. R.; Montgomery, J. A. Jr.; Vreven, T.; Kudin, K. N.; Burant, J. C.; Millam, J. M.; Iyengar, S. S.; Tomasi, J.; Barone, V.; Mennucci, B.; Cossi, M.; Scalmani, G.; Rega, N.; Petersson, G. A.; Nakatsuji, H.; Hada, M.; Ehara, M.; Toyota, K.; Fukuda, R.; Hasegawa, J.; Ishida, M.; Nakajima, T.; Honda, Y.; Kitao, O.; Nakai, H.; Klene, M.; Li, X.; Knox, J. E.; Hratchian, H. P.; Cross, J. B.; Adamo, C.; Jaramillo, J.; Gomperts, R.; Stratmann, R. E.; Yazyev, O.; Austin, A. J.; Cammi, R.; Pomelli, C.; Ochterski, J. W.; Ayala, P. Y.; Morokuma, K.; Voth, G. A.; Salvador, P.; Dannenberg, J. J.; Zakrzewski, V. G.; Dapprich, S.; Daniels, A. D.; Strain, M. C.; Farkas, O.; Malick, D. K.; Rabuck, A. D.; Raghavachari, K.; Foresman, J. B.; Ortiz, J. V.; Cui, Q.; Baboul, A. G.; Clifford, S.; Cioslowski, J.; Stefanov, B. B.; Liu, G.; Liashenko, A.; Piskorz, P.; Komaromi, I.; Martin, R. L.; Fox, D. J.; Keith, T.; Al-Laham, M. A.; Peng, C. Y.; Nanayakkara, A.; Challacombe, M.; Gill, P. M. W.; Johnson, B.; Chen, W.; Wong, M. W.; Gonzalez, C.; Pople, J. A. Gaussian 03, revision B. 04; Gaussian, Inc.: Pittsburgh, PA, 2003.

\footnotetext{
${ }^{\dagger}$ University of California, Los Angeles

${ }^{\star}$ Brock University
} 


\section{B. Molecular Geometries and Energies}

A note concerning the DA reaction: Contrary to previously reported theoretical studies involving acrolein as dienophile, ${ }^{3}$ the most stable transition structure corresponds to TS11, where the acrolein fragment is in a $s$-trans-endo disposition. TS11 presents two stabilizing $\mathrm{C}-\mathrm{H} \cdots \mathrm{O}$ electrostatic interactions between the methyl groups attached to the nitrogen atom of the diene and the oxygen atom of the carbonyl group of the acrolein. The corresponding $s$-cis-endo transition structure, in which no $\mathrm{C}-\mathrm{H} \cdots \mathrm{O}$ stabilizing interactions can be established, was found to be $3.5 \mathrm{kcal} / \mathrm{mol}$ higher in energy than TS11.

Cartesian coordinates and energies for all relevant structures are presented below.

\begin{tabular}{lrrr} 
& \multicolumn{3}{c}{ Acrolein } \\
$\mathrm{C}$ & 1.208403 & 1.293539 & 0.000000 \\
$\mathrm{H}$ & 2.116295 & 0.692688 & 0.000000 \\
$\mathrm{H}$ & 1.339679 & 2.371480 & 0.000000 \\
$\mathrm{C}$ & 0.000000 & 0.719028 & 0.000000 \\
$\mathrm{H}$ & -0.920483 & 1.298455 & 0.000000 \\
$\mathrm{C}$ & -0.147045 & -0.748748 & 0.000000 \\
$\mathrm{H}$ & 0.814703 & -1.310894 & 0.000000 \\
$\mathrm{O}$ & -1.214793 & -1.329330 & 0.000000
\end{tabular}

Energies at $0 \mathrm{~K}$ and also H and G @ $298.15 \mathrm{~K}$ :

$\mathrm{E}(0 \mathrm{~K}), \mathrm{E}+\mathrm{ZPE}(0 \mathrm{~K})$, Enthalpy, Free Energy

$-191.911974,-191.850317,-191.845020,-191.876596$

\begin{tabular}{lrrr} 
& \multicolumn{3}{c}{ Diene 5 } \\
C & 2.408230 & 1.523163 & 0.151090 \\
C & 1.516140 & 0.517438 & 0.033671 \\
O & 1.854290 & -0.808827 & -0.070316 \\
C & 3.229866 & -1.141061 & -0.050715 \\
C & 0.073116 & 0.729189 & 0.011262 \\
C & -0.824758 & -0.277065 & -0.100413 \\
N & -2.196050 & -0.164887 & -0.215402 \\
C & -3.007737 & -1.286004 & 0.229588 \\
C & -2.803765 & 1.145708 & -0.094925 \\
H & 3.479603 & 1.374644 & 0.174077 \\
H & 2.050611 & 2.543168 & 0.228393 \\
H & -0.245042 & 1.763446 & 0.087486 \\
H & -0.464241 & -1.300523 & -0.143647 \\
H & 3.703851 & -0.830904 & 0.890161 \\
H & 3.282174 & -2.228076 & -0.143895 \\
H & 3.766037 & -0.676090 & -0.888813 \\
H & -3.863051 & 1.077769 & -0.359699 \\
H & -2.724016 & 1.562060 & 0.923949 \\
H & -2.320889 & 1.842650 & -0.787478 \\
H & -2.506220 & -2.223920 & -0.026743 \\
H & -3.189215 & -1.281145 & 1.317928 \\
H & -3.978117 & -1.266474 & -0.278730
\end{tabular}

Energies at 0K and also H and G @ 298.15 K:

$\mathrm{E}(0 \mathrm{~K}), \mathrm{E}+\mathrm{ZPE}(0 \mathrm{~K})$, Enthalpy, Free Energy

$-404.493422,-404.300192,-404.288394,-404.336442$

\begin{tabular}{|c|c|c|c|}
\hline \multicolumn{4}{|c|}{$\begin{array}{c}\text { TS1 - DA uncatalyzed } \\
\text { TS1 }\end{array}$} \\
\hline & -1.157628 & 2.084187 & 0.3089 \\
\hline$e^{2}$ & & & \\
\hline$C$ & 0.900295 & & 7807 \\
\hline $\mathrm{O}$ & 2.121812 & & \\
\hline $\mathrm{C}$ & -1.903372 & 0.4 & \\
\hline $\mathrm{C}$ & -1.6 & -0.6 & \\
\hline$C^{-1}$ & -2.5 & & \\
\hline C & -3.935129 & -0.7 & \\
\hline C & -0.360353 & -1.1 & \\
\hline 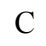 & 0.7 & -0 . & \\
\hline $\mathrm{N}$ & 2.0 & -1 . & \\
\hline $\mathrm{C}$ & 3.142 & -0. & \\
\hline $\mathrm{C}$ & 2.378 & & \\
\hline $\mathrm{H}$ & -0.288 & -1.8 & \\
\hline & 3.95 & -1. & \\
\hline $\mathrm{H}$ & 3.43 & & \\
\hline$H$ & 2.812 & -0.1 & \\
\hline & 1.757134 & -2.6 & -1 . \\
\hline & 2.240 & -1. & \\
\hline & 3.42 & -2.0 & \\
\hline $\mathrm{H}$ & -4.067 & & \\
\hline & -4.490903 & -1.2 & -1 \\
\hline & -4.319623 & -0.9 & \\
\hline & 0.67 & -0.1 & \\
\hline & -2.936991 & & 69 \\
\hline 11 & -1.278872 & 0.4 & 3069 \\
\hline $\mathrm{H}$ & 0.865513 & 2.35 & 1.033070 \\
\hline & -1.60 & 2.7 & \\
\hline & -1.73 & & -0. \\
\hline & 0.203910 & 1.464977 & 106440 \\
\hline
\end{tabular}

Energies at 0K and also H and G @ 298.15 K:

$\mathrm{E}(0 \mathrm{~K}), \mathrm{E}+\mathrm{ZPE}(0 \mathrm{~K})$, Enthalpy, Free Energy

$-596.389415,-596.131539,-596.115521,-596.173027$

\begin{tabular}{rrrr}
\multicolumn{4}{c}{ DA uncatalyzed } \\
product \\
C & 0.795695 & 1.050325 & 0.426483 \\
C & 0.505127 & -0.515748 & 0.543140 \\
C & -0.745922 & -0.919224 & -0.200341 \\
C & -1.849217 & -0.170630 & -0.095853 \\
C & -1.804187 & 1.115585 & 0.692827 \\
C & -0.513574 & 1.888642 & 0.339922 \\
O & -2.993554 & -0.510498 & -0.780901 \\
C & -4.177000 & -0.622068 & 0.002425 \\
N & 1.611882 & -1.432678 & 0.283711 \\
C & 2.051616 & -1.609300 & -1.094902 \\
C & 1.653922 & 1.416702 & -0.765155 \\
O & 2.769943 & 1.886680 & -0.693941 \\
C & 2.731581 & -1.290727 & 1.203506 \\
H & -0.765086 & -1.859062 & -0.741736 \\
H & 3.394672 & -2.157866 & 1.099920 \\
H & 3.336812 & -0.382472 & 1.037374 \\
H & 2.361258 & -1.273031 & 2.235421 \\
H & 2.612274 & -2.549532 & -1.168815 \\
H & 1.189163 & -1.683218 & -1.762800 \\
H & 2.709102 & -0.806605 & -1.469369 \\
H & -4.423800 & 0.313975 & 0.521100 \\
H & -4.983893 & -0.862492 & -0.693325 \\
H & -4.085595 & -1.427020 & 0.744745
\end{tabular}




$$
\begin{array}{rrrr}
\mathrm{H} & 0.297278 & -0.641239 & 1.617776 \\
\mathrm{H} & -2.671568 & 1.742607 & 0.460757 \\
\mathrm{H} & -1.834447 & 0.913475 & 1.773895 \\
\mathrm{H} & 1.369826 & 1.340697 & 1.311997 \\
\mathrm{H} & -0.420174 & 2.775496 & 0.975824 \\
\mathrm{H} & -0.637632 & 2.258528 & -0.686738 \\
\mathrm{H} & 1.169273 & 1.255700 & -1.755586
\end{array}
$$

\begin{tabular}{|c|c|c|c|}
\hline \multicolumn{4}{|c|}{$\begin{array}{l}\text { DA 1-MeOH catalyzed } \\
\text { starting complex }\end{array}$} \\
\hline $\mathrm{C}$ & 0.483555 & -2.856548 & 0.552329 \\
\hline $\mathrm{C}$ & -0.632925 & -2.205247 & 0.194173 \\
\hline $\mathrm{C}$ & -0.697242 & -1.476462 & -1.074104 \\
\hline $\mathrm{O}$ & -1.681487 & -0.855178 & -1.467449 \\
\hline $\mathrm{O}$ & -3.649396 & -0.598012 & 0.547617 \\
\hline $\mathrm{C}$ & -4.905489 & -0.891967 & -0.035406 \\
\hline $\mathrm{C}$ & 2.719550 & -0.491145 & 1.434485 \\
\hline $\mathrm{C}$ & 2.612606 & 0.204898 & 0.279343 \\
\hline $\mathrm{C}$ & 1.459881 & 0.969144 & -0.189652 \\
\hline $\mathrm{C}$ & 0.446810 & 1.397824 & 0.611444 \\
\hline $\mathrm{N}$ & -0.621024 & 2.168998 & 0.256508 \\
\hline $\mathrm{C}$ & -0.817314 & 2.532446 & -1.137184 \\
\hline $\mathrm{O}$ & 3.610141 & 0.268712 & -0.666133 \\
\hline $\mathrm{C}$ & 4.806046 & -0.445305 & -0.418190 \\
\hline $\mathrm{C}$ & -1.772919 & 2.267055 & 1.146524 \\
\hline $\mathrm{H}$ & 1.488218 & 1.234316 & -1.241217 \\
\hline $\mathrm{H}$ & -2.218468 & 3.264916 & 1.061730 \\
\hline $\mathrm{H}$ & -2.541780 & 1.512351 & 0.929268 \\
\hline $\mathrm{H}$ & -1.441192 & 2.132139 & 2.180335 \\
\hline $\mathrm{H}$ & 0.094766 & 2.992498 & -1.534452 \\
\hline $\mathrm{H}$ & -1.074477 & 1.663985 & -1.758922 \\
\hline $\mathrm{H}$ & -1.626260 & 3.264603 & -1.203149 \\
\hline $\mathrm{H}$ & 4.613312 & -1.518968 & -0.285306 \\
\hline $\mathrm{H}$ & 5.438518 & -0.293367 & -1.295542 \\
\hline $\mathrm{H}$ & 5.323663 & -0.066984 & 0.473521 \\
\hline $\mathrm{H}$ & 0.465050 & 1.149025 & 1.669865 \\
\hline $\mathrm{H}$ & 3.631244 & -0.983227 & 1.746377 \\
\hline $\mathrm{H}$ & 1.884193 & -0.531044 & 2.122345 \\
\hline $\mathrm{H}$ & -1.522530 & -2.177310 & 0.820154 \\
\hline $\mathrm{H}$ & 0.557478 & -3.400481 & 1.489226 \\
\hline $\mathrm{H}$ & 1.366844 & -2.856540 & -0.079877 \\
\hline $\mathrm{H}$ & 0.212397 & -1.522765 & -1.702071 \\
\hline $\mathrm{H}$ & -2.967732 & -0.687037 & -0.150337 \\
\hline $\mathrm{H}$ & -5.661098 & -0.803036 & 0.751545 \\
\hline $\mathrm{H}$ & -5.170633 & -0.193107 & -0.844105 \\
\hline $\mathrm{H}$ & -4.953772 & -1.915291 & -0.439799 \\
\hline
\end{tabular}

Energies at $0 \mathrm{~K}$ and also $\mathrm{H}$ and $\mathrm{G} @ 298.15 \mathrm{~K}$ :

$\mathrm{E}(0 \mathrm{~K}), \mathrm{E}+\mathrm{ZPE}(0 \mathrm{~K})$, Enthalpy, Free Energy

$-596.427327,-596.165263,-596.150059,-596.206541$

Energies at $0 \mathrm{~K}$ and also H and G @ 298.15 K:

$\mathrm{E}(0 \mathrm{~K}), \mathrm{E}+\mathrm{ZPE}(0 \mathrm{~K})$, Enthalpy, Free Energy

$-712.136655,-711.826906,-711.804179,-711.882318$

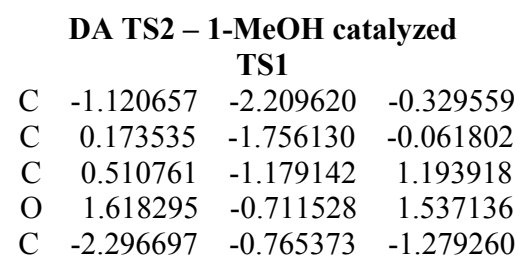

$\begin{array}{lrrr}\mathrm{C} & -2.424379 & 0.259494 & -0.332194 \\ \mathrm{O} & -3.499629 & 0.353866 & 0.503860 \\ \mathrm{C} & -4.646018 & -0.448072 & 0.257236 \\ \mathrm{C} & -1.394754 & 1.156907 & 0.034413 \\ \mathrm{C} & -0.186877 & 1.244904 & -0.636136 \\ \mathrm{~N} & 0.879612 & 1.965291 & -0.264167 \\ \mathrm{C} & 2.077155 & 2.030859 & -1.102456 \\ \mathrm{C} & 0.928221 & 2.600846 & 1.048432 \\ \mathrm{O} & 3.464810 & -0.768679 & -0.477031 \\ \mathrm{C} & 4.595257 & -1.528060 & -0.103452 \\ \mathrm{H} & -1.573427 & 1.749744 & 0.924716 \\ \mathrm{H} & 2.412979 & 3.071082 & -1.181497 \\ \mathrm{H} & 2.877961 & 1.399763 & -0.702114 \\ \mathrm{H} & 1.834709 & 1.672075 & -2.105174 \\ \mathrm{H} & 0.187670 & 3.407776 & 1.123779 \\ \mathrm{H} & 0.746785 & 1.860612 & 1.833245 \\ \mathrm{H} & 1.921968 & 3.025845 & 1.197375 \\ \mathrm{H} & -4.428051 & -1.516410 & 0.375671 \\ \mathrm{H} & -5.382667 & -0.147519 & 1.004531 \\ \mathrm{H} & -5.051372 & -0.271452 & -0.747025 \\ \mathrm{H} & -0.059345 & 0.723857 & -1.578628 \\ \mathrm{H} & -3.178850 & -1.321912 & -1.577950 \\ \mathrm{H} & -1.598103 & -0.605846 & -2.092085 \\ \mathrm{H} & 0.953245 & -1.804008 & -0.819268 \\ \mathrm{H} & -1.272276 & -2.914419 & -1.143438 \\ \mathrm{H} & -1.788374 & -2.367591 & 0.515293 \\ \mathrm{H} & -0.312264 & -1.166911 & 1.939969 \\ \mathrm{H} & 2.833338 & -0.766277 & 0.286149 \\ \mathrm{H} & 5.283126 & -1.549022 & -0.956281 \\ \mathrm{H} & 5.134848 & -1.095813 & 0.755294 \\ \mathrm{H} & 4.339719 & -2.569555 & 0.150043\end{array}$

Energies at $0 \mathrm{~K}$ and also H and G @ $298.15 \mathrm{~K}$ :

$\mathrm{E}(0 \mathrm{~K}), \mathrm{E}+\mathrm{ZPE}(0 \mathrm{~K})$, Enthalpy, Free Energy

$-712.125397,-711.813857,-711.793156,-711.863641$

\begin{tabular}{lrrr}
\multicolumn{4}{c}{ DA 1-MeOH catalyzed } \\
product complex \\
C & 0.381707 & -0.563140 & -0.040693 \\
C & -0.337859 & 0.775437 & -0.511906 \\
C & -1.734078 & 0.859468 & 0.059421 \\
C & -2.534586 & -0.208455 & -0.029635 \\
C & -2.007137 & -1.470048 & -0.669884 \\
C & -0.584206 & -1.792222 & -0.146835 \\
O & -3.795789 & -0.193828 & 0.520116 \\
C & -4.863557 & -0.526949 & -0.361087 \\
N & 0.391077 & 2.029595 & -0.368620 \\
C & 0.642032 & 2.513402 & 0.981706 \\
C & 0.855435 & -0.514049 & 1.388679 \\
O & 2.003922 & -0.698377 & 1.759133 \\
C & 1.569325 & 2.143004 & -1.222385 \\
H & -2.092447 & 1.792602 & 0.480518 \\
H & 1.911728 & 3.184686 & -1.215539 \\
H & 2.415713 & 1.502662 & -0.922761 \\
H & 1.299123 & 1.887070 & -2.253574 \\
H & 0.895495 & 3.579524 & 0.935330 \\
H & -0.257504 & 2.413542 & 1.596236 \\
H & 1.472126 & 2.003048 & 1.500058 \\
H & -4.758543 & -1.536251 & -0.780884 \\
H & -5.777359 & -0.487285 & 0.235801 \\
H & -4.935984 & 0.196015 & -1.185208 \\
H & -0.429262 & 0.631805 & -1.599920
\end{tabular}




$$
\begin{array}{lrrr}
\mathrm{H} & -2.663534 & -2.320324 & -0.458043 \\
\mathrm{H} & -1.975912 & -1.356393 & -1.763550 \\
\mathrm{H} & 1.261227 & -0.723136 & -0.670166 \\
\mathrm{H} & -0.121345 & -2.555341 & -0.780766 \\
\mathrm{H} & -0.701307 & -2.253670 & 0.842586 \\
\mathrm{H} & 0.061697 & -0.344203 & 2.146053 \\
\mathrm{H} & 3.273804 & -0.713792 & 0.349602 \\
\mathrm{O} & 3.711299 & -0.657086 & -0.523009 \\
\mathrm{C} & 4.859983 & -1.482566 & -0.506753 \\
\mathrm{H} & 4.620634 & -2.539295 & -0.306046 \\
\mathrm{H} & 5.320694 & -1.422649 & -1.497854 \\
\mathrm{H} & 5.605594 & -1.154736 & 0.234767
\end{array}
$$

\begin{tabular}{|c|c|c|c|}
\hline \multicolumn{4}{|c|}{$\begin{array}{c}\text { DA 2-MeOH catalyzed (cooperative mode) } \\
\text { starting complex }\end{array}$} \\
\hline $\mathrm{C}$ & 0.920716 & -2.347553 & -1.468133 \\
\hline $\mathrm{C}$ & -0.155643 & -1.546022 & -1.400494 \\
\hline $\mathrm{C}$ & -0.214729 & -0.321924 & -2.188054 \\
\hline $\mathrm{O}$ & -1.159880 & 0.467647 & -2.211908 \\
\hline $\mathrm{O}$ & -3.231291 & 0.070837 & -0.419104 \\
\hline $\mathrm{C}$ & -4.303042 & -0.485590 & -1.167085 \\
\hline $\mathrm{O}$ & -1.856132 & -1.247230 & 1.597590 \\
\hline $\mathrm{C}$ & -2.682580 & -2.022618 & 2.445954 \\
\hline $\mathrm{C}$ & 2.513149 & -1.275389 & 0.998060 \\
\hline $\mathrm{C}$ & 2.758253 & -0.063383 & 0.445150 \\
\hline $\mathrm{O}$ & 3.998372 & 0.332135 & -0.003538 \\
\hline $\mathrm{C}$ & 5.070956 & -0.580765 & 0.123091 \\
\hline $\mathrm{C}$ & 1.799321 & 1.007986 & 0.211538 \\
\hline $\mathrm{C}$ & 0.559081 & 1.062730 & 0.771855 \\
\hline $\mathrm{N}$ & -0.399996 & 2.007423 & 0.542279 \\
\hline $\mathrm{C}$ & -1.486919 & 2.169360 & 1.498834 \\
\hline $\mathrm{C}$ & -0.118432 & 3.129972 & -0.332185 \\
\hline $\mathrm{H}$ & 2.166536 & 1.817374 & -0.410858 \\
\hline $\mathrm{H}$ & -1.267440 & 2.950029 & 2.244658 \\
\hline $\mathrm{H}$ & -2.408598 & 2.433216 & 0.970750 \\
\hline $\mathrm{H}$ & -1.653158 & 1.220733 & 2.013245 \\
\hline $\mathrm{H}$ & 0.612144 & 3.828914 & 0.106499 \\
\hline $\mathrm{H}$ & 0.272167 & 2.768897 & -1.287985 \\
\hline $\mathrm{H}$ & -1.047191 & 3.672472 & -0.526448 \\
\hline $\mathrm{H}$ & 4.869277 & -1.516851 & -0.416066 \\
\hline $\mathrm{H}$ & 5.942773 & -0.088604 & -0.313763 \\
\hline $\mathrm{H}$ & 5.276493 & -0.818072 & 1.175744 \\
\hline $\mathrm{H}$ & 0.235228 & 0.282932 & 1.455825 \\
\hline $\mathrm{H}$ & 3.293675 & -1.994874 & 1.208016 \\
\hline $\mathrm{H}$ & 1.510756 & -1.533094 & 1.314275 \\
\hline $\mathrm{H}$ & -0.979693 & -1.756007 & -0.723828 \\
\hline $\mathrm{H}$ & 0.994734 & -3.262291 & -0.889937 \\
\hline $\mathrm{H}$ & 1.759775 & -2.115771 & -2.119623 \\
\hline $\mathrm{H}$ & 0.670316 & -0.123518 & -2.823838 \\
\hline $\mathrm{H}$ & -2.495974 & 0.275082 & -1.045383 \\
\hline $\mathrm{H}$ & -5.110413 & -0.717323 & -0.466373 \\
\hline $\mathrm{H}$ & -4.685971 & 0.225024 & -1.912549 \\
\hline $\mathrm{H}$ & -4.015978 & -1.412185 & -1.685818 \\
\hline $\mathrm{H}$ & -2.425561 & -0.804506 & 0.926485 \\
\hline $\mathrm{H}$ & -3.426570 & -1.413876 & 2.983417 \\
\hline $\mathrm{H}$ & -3.219385 & -2.816649 & 1.902580 \\
\hline $\mathrm{H}$ & -2.037303 & -2.500944 & 3.189509 \\
\hline
\end{tabular}

Energies at 0K and also H and G @ 298.15 K:

$\mathrm{E}(0 \mathrm{~K}), \mathrm{E}+\mathrm{ZPE}(0 \mathrm{~K})$, Enthalpy, Free Energy

$-712.156448,-711.840577,-711.820759,-711.889672$

Energies at 0K and also H and G @ 298.15 K:
$\mathrm{E}(0 \mathrm{~K}), \mathrm{E}+\mathrm{ZPE}(0 \mathrm{~K})$, Enthalpy, Free Energy $-827.870838,-827.506787,-827.479986$, $-827.566413$

$\Delta \mathrm{G}(\mathrm{solv})(\mathrm{PhMe})(\mathrm{kcal} / \mathrm{mol})=6.20$

DA TS3 - 2-MeOH catalyzed (cooperative mode) TS1

$\begin{array}{llll}\text { C } & 1.226716 & -1.892217 & -1.247353\end{array}$

$\begin{array}{llll}\text { C } & 0.005982 & -1.233423 & -1.361347\end{array}$

$\begin{array}{llll}\text { C } & -0.125817 & 0.013525 & -2.026414\end{array}$

$\begin{array}{llll}\mathrm{O} & -1.161235 & 0.716963 & -2.105947\end{array}$

$\begin{array}{llll}\text { C } & 2.130473 & -1.437202 & 0.635100\end{array}$

$\begin{array}{llll}\text { C } & 2.603500 & -0.136902 & 0.452548\end{array}$

$\begin{array}{llll}\text { O } & 3.887790 & 0.128532 & 0.065498\end{array}$

$\begin{array}{llll}\text { C } & 4.844221 & -0.920452 & 0.066913\end{array}$

$\begin{array}{llll}\text { C } & 1.806559 & 1.035234 & 0.438242\end{array}$

$\begin{array}{llll}\text { C } & 0.475077 & 1.069299 & 0.810256\end{array}$

$\mathrm{N} \quad-0.344531 \quad 2.123735 \quad 0.667164$

$\begin{array}{llll}\text { C } & -1.681990 & 2.130388 & 1.251855\end{array}$

$\begin{array}{llll}\text { C } & 0.083288 & 3.316615 & -0.047546\end{array}$

$\begin{array}{llll}\text { O } & -3.174650 & -0.208596 & -0.593036\end{array}$

$\begin{array}{llll}\text { C } & -4.068727 & -0.936218 & -1.419602\end{array}$

$\begin{array}{llll}\text { O } & -1.842612 & -1.200099 & 1.606778\end{array}$

$\begin{array}{llll}\text { C } & -2.665930 & -1.983984 & 2.449901\end{array}$

$\begin{array}{llll}\mathrm{H} & 2.300802 & 1.932213 & 0.080668\end{array}$

$\begin{array}{llll}\mathrm{H} & -1.768759 & 2.930444 & 1.999381\end{array}$

$\begin{array}{llll}\mathrm{H} & -2.436155 & 2.282164 & 0.473081\end{array}$

$\begin{array}{llll}\mathrm{H} & -1.871614 & 1.168159 & 1.729037\end{array}$

$\begin{array}{llll}\mathrm{H} & 0.811907 & 3.899135 & 0.533460\end{array}$

$\mathrm{H} \quad 0.531284 \quad 3.038650 \quad-1.006488$

$\begin{array}{llll}\mathrm{H} & -0.788527 & 3.943694 & -0.243180\end{array}$

H $\quad 4.592153 \quad-1.703040 \quad-0.659390$

$\begin{array}{llll}\mathrm{H} & 5.791188 & -0.457988 & -0.218044\end{array}$

$\begin{array}{llll}\mathrm{H} & 4.943804 & -1.372298 & 1.062045\end{array}$

$\begin{array}{llll}\mathrm{H} & 0.005244 & 0.205533 & 1.271881\end{array}$

$\begin{array}{llll}\mathrm{H} & 2.836747 & -2.249376 & 0.768589\end{array}$

$\begin{array}{llll}\mathrm{H} & 1.198715 & -1.567083 & 1.171571\end{array}$

$\mathrm{H} \quad-0.865347 \quad-1.632363 \quad-0.849730$

$\mathrm{H} \quad 1.237214 \quad-2.949943 \quad-0.999451$

$\mathrm{H} \quad 2.053973 \quad-1.573578 \quad-1.878531$

$\begin{array}{llll}\mathrm{H} & 0.786336 & 0.376405 & -2.542319\end{array}$

$\begin{array}{llll}\mathrm{H} & -2.445602 & 0.157727 & -1.174079\end{array}$

H $\quad-4.860606 \quad-1.344528 \quad-0.783422$

$\mathrm{H} \quad-4.534835 \quad-0.289115 \quad-2.176003$

$\mathrm{H} \quad-3.574661 \quad-1.771589 \quad-1.937979$

$\mathrm{H} \quad-2.371605 \quad-0.921850 \quad 0.818918$

$\begin{array}{llll}\mathrm{H} & -3.561812 & -1.440703 & 2.788574\end{array}$

$\mathrm{H} \quad-2.994306 \quad-2.917152 \quad 1.965686$

H $\quad-2.078277 \quad-2.252041 \quad 3.333896$

Energies at $0 \mathrm{~K}$ and also $\mathrm{H}$ and $\mathrm{G} @ 298.15 \mathrm{~K}$ : $\mathrm{E}(0 \mathrm{~K}), \mathrm{E}+\mathrm{ZPE}(0 \mathrm{~K})$, Enthalpy, Free Energy $-827.863562,-827.497683,-827.472837$, $-827.552963$

$\Delta \mathrm{G}(\mathrm{solv})(\mathrm{PhMe})(\mathrm{kcal} / \mathrm{mol})=3.61$

DA 2-MeOH catalyzed (cooperative mode) intermediate

$\begin{array}{llll}\text { C } & -4.916879 & -1.047024 & 0.048445\end{array}$

$\begin{array}{llll}\mathrm{O} & -4.005068 & 0.017208 & -0.198850\end{array}$

$\begin{array}{llll}\text { C } & -2.677417 & -0.229341 & -0.370433\end{array}$ 


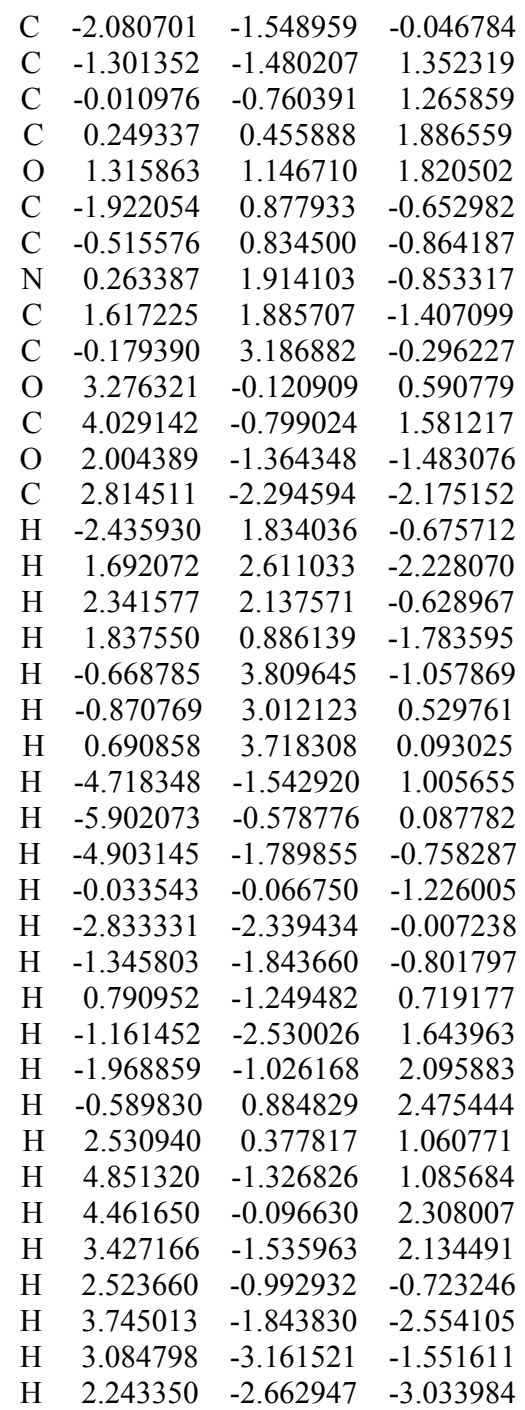

$\mathrm{E}(0 \mathrm{~K}), \mathrm{E}+\mathrm{ZPE}(0 \mathrm{~K})$, Enthalpy, Free Energy $-827.876832,-827.508250,-827.483801$, $-827.562772$

$\Delta \mathrm{G}(\mathrm{solv})(\mathrm{PhMe})(\mathrm{kcal} / \mathrm{mol})=0.59$

\section{DA 2-MeOH catalyzed (cooperative mode)}

$$
\text { TS2 }
$$

$\begin{array}{lrrr}\text { C } & -5.057418 & -1.077523 & 0.055392 \\ \text { O } & -4.136448 & -0.014654 & -0.146583 \\ \mathrm{C} & -2.795605 & -0.275272 & -0.221921 \\ \mathrm{C} & -2.195802 & -1.521048 & 0.349644 \\ \mathrm{C} & -1.241142 & -1.149931 & 1.541506 \\ \mathrm{C} & -0.030679 & -0.372887 & 1.120466 \\ \mathrm{C} & 0.346650 & 0.817725 & 1.781710 \\ \mathrm{O} & 1.423166 & 1.442229 & 1.626016 \\ \mathrm{C} & -2.008934 & 0.726945 & -0.680170 \\ \mathrm{C} & -0.563352 & 0.597751 & -0.792616 \\ \mathrm{~N} & 0.199530 & 1.686680 & -1.060317 \\ \mathrm{C} & 1.478638 & 1.572700 & -1.750820 \\ \mathrm{C} & -0.206155 & 3.026066 & -0.664946 \\ \mathrm{O} & 3.423229 & 0.035637 & 0.491658 \\ \mathrm{C} & 4.229884 & -0.431511 & 1.560622\end{array}$

$\begin{array}{rrrr}\mathrm{O} & 2.104239 & -1.643222 & -1.243357 \\ \mathrm{C} & 2.900016 & -2.691159 & -1.761540 \\ \mathrm{H} & -2.479160 & 1.665922 & -0.956415 \\ \mathrm{H} & 1.444457 & 2.121260 & -2.703330 \\ \mathrm{H} & 2.286612 & 1.982532 & -1.136211 \\ \mathrm{H} & 1.698495 & 0.523015 & -1.952565 \\ \mathrm{H} & -0.732776 & 3.550970 & -1.476637 \\ \mathrm{H} & -0.862348 & 2.980678 & 0.207266 \\ \mathrm{H} & 0.680100 & 3.604773 & -0.392389 \\ \mathrm{H} & -5.007839 & -1.480799 & 1.074664 \\ \mathrm{H} & -6.046186 & -0.640642 & -0.099986 \\ \mathrm{H} & -4.908736 & -1.892154 & -0.664582 \\ \mathrm{H} & -0.167863 & -0.310458 & -1.236020 \\ \mathrm{H} & -2.956804 & -2.223832 & 0.696102 \\ \mathrm{H} & -1.600516 & -2.047133 & -0.406943 \\ \mathrm{H} & 0.771025 & -0.951315 & 0.667480 \\ \mathrm{H} & -0.946580 & -2.095990 & 2.015655 \\ \mathrm{H} & -1.824094 & -0.591281 & 2.285418 \\ \mathrm{H} & -0.432279 & 1.285907 & 2.420861 \\ \mathrm{H} & 2.683109 & 0.579746 & 0.884112 \\ \mathrm{H} & 5.053371 & -1.011978 & 1.132400 \\ \mathrm{H} & 4.658349 & 0.401543 & 2.135317 \\ \mathrm{H} & 3.673532 & -1.078951 & 2.255243 \\ \mathrm{H} & 2.654536 & -1.103769 & -0.623526 \\ \mathrm{H} & 3.786824 & -2.321306 & -2.299657 \\ \mathrm{H} & 3.241448 & -3.387831 & -0.979143 \\ \mathrm{H} & 2.286505 & -3.256712 & -2.470745\end{array}$

$\mathrm{E}(0 \mathrm{~K}), \mathrm{E}+\mathrm{ZPE}(0 \mathrm{~K})$, Enthalpy, Free Energy $-827.875333,-827.506930,-827.483007$, $-827.561457$

$\Delta \mathrm{G}(\mathrm{solv})(\mathrm{PhMe})(\mathrm{kcal} / \mathrm{mol})=1.84$

\section{DA 2-MeOH catalyzed (cooperative mode)} product complex

$\begin{array}{lrrr}\mathrm{C} & -1.249750 & 0.367215 & -1.952801 \\ \mathrm{C} & -0.068845 & -0.117567 & -1.081661 \\ \mathrm{C} & -0.455698 & -0.254438 & 0.472197 \\ \mathrm{C} & -1.931772 & -0.512589 & 0.680424 \\ \mathrm{C} & -2.838141 & 0.142482 & -0.053095 \\ \mathrm{C} & -2.381749 & 1.059909 & -1.161073 \\ \mathrm{C} & 0.501613 & -1.396710 & -1.633302 \\ \mathrm{O} & 1.675979 & -1.719081 & -1.577002 \\ \mathrm{~N} & 0.465687 & -1.138651 & 1.188981 \\ \mathrm{C} & 0.090264 & -2.544702 & 1.251003 \\ \mathrm{O} & -4.183448 & -0.109127 & 0.109043 \\ \mathrm{C} & -5.002162 & 1.031315 & 0.342857 \\ \mathrm{C} & 0.870357 & -0.631470 & 2.497237 \\ \mathrm{O} & 3.454353 & -0.202904 & 0.059127 \\ \mathrm{C} & 4.484526 & -0.127762 & -0.917311 \\ \mathrm{O} & 1.913660 & 2.035474 & 0.584961 \\ \mathrm{C} & 2.672424 & 3.225118 & 0.672061 \\ \mathrm{H} & -2.257803 & -1.188942 & 1.464353 \\ \mathrm{H} & 0.051202 & -0.634159 & 3.241773 \\ \mathrm{H} & 1.679504 & -1.258811 & 2.887503 \\ \mathrm{H} & 1.249574 & 0.387865 & 2.390249 \\ \mathrm{H} & -0.724612 & -2.757274 & 1.966875 \\ \mathrm{H} & -0.232903 & -2.905256 & 0.269739 \\ \mathrm{H} & 0.962087 & -3.135355 & 1.553869 \\ \mathrm{H} & -4.954552 & 1.755753 & -0.481304 \\ \mathrm{H} & -6.026631 & 0.661845 & 0.427784 \\ \mathrm{H} & -4.719716 & 1.536671 & 1.276793\end{array}$




$\begin{array}{rrrr}\mathrm{H} & -0.230697 & 0.751336 & 0.842124 \\ \mathrm{H} & -3.209971 & 1.285604 & -1.841177 \\ \mathrm{H} & -2.021684 & 2.018557 & -0.760688 \\ \mathrm{H} & 0.741841 & 0.616691 & -1.086347 \\ \mathrm{H} & -0.876229 & 1.040703 & -2.732670 \\ \mathrm{H} & -1.697591 & -0.491662 & -2.470849 \\ \mathrm{H} & -0.224188 & -2.085748 & -2.118919 \\ \mathrm{H} & 2.754288 & -0.790230 & -0.297973 \\ \mathrm{H} & 5.250411 & 0.552279 & -0.533256 \\ \mathrm{H} & 4.947067 & -1.109056 & -1.090315 \\ \mathrm{H} & 4.121663 & 0.258902 & -1.880433 \\ \mathrm{H} & 2.530880 & 1.279400 & 0.444462 \\ \mathrm{H} & 3.387013 & 3.210364 & 1.510130 \\ \mathrm{H} & 3.234962 & 3.438263 & -0.251579 \\ \mathrm{H} & 1.975512 & 4.053126 & 0.838745\end{array}$

$\mathrm{E}(0 \mathrm{~K}), \mathrm{E}+\mathrm{ZPE}(0 \mathrm{~K})$, Enthalpy, Free Energy $-827.889258,-827.519514,-827.495261$, $-827.575537$

$\Delta \mathrm{G}(\mathrm{solv})(\mathrm{PhMe})(\mathrm{kcal} / \mathrm{mol})=2.73$

DA 2-MeOH catalyzed (bifurcated mode) starting material complex

C $\quad \begin{array}{llll}\text { C } & 1.706770 & 2.327813 & 1.127433\end{array}$

$\begin{array}{llll}\text { C } & 0.389613 & 2.165447 & 0.919410\end{array}$

C $\quad-0.291173 \quad 0.972554 \quad 1.401529$

$\begin{array}{llll}\mathrm{O} & -1.491273 & 0.744306 & 1.211289\end{array}$

$\begin{array}{llll}\mathrm{O} & -2.653519 & -1.850104 & 1.280989\end{array}$

C $\quad-3.716058 \quad-1.920724 \quad 2.212250$

$\begin{array}{llll}\text { O } & -2.239497 & 2.392539 & -0.997687\end{array}$

C $\quad-3.531780 \quad 2.971718 \quad-1.045561$

C $\quad 0.567898 \quad-0.763445 \quad-1.239253$

$\begin{array}{llll}\mathrm{N} & -0.512658 & -1.536000 & -1.530315\end{array}$

$\begin{array}{llll}\text { C } & -1.780326 & -0.938423 & -1.934935\end{array}$

$\begin{array}{llll}\text { C } & 1.674609 & -1.132797 & -0.530347\end{array}$

$\begin{array}{llll}\text { C } & 2.872922 & -0.317415 & -0.412536\end{array}$

$\begin{array}{llll}\text { O } & 3.570420 & -0.674584 & 0.724169\end{array}$

$\begin{array}{llll}\text { C } & 4.881867 & -0.161658 & 0.884306\end{array}$

$\begin{array}{llll}\text { C } & 3.279935 & 0.658114 & -1.257408\end{array}$

$\begin{array}{llll}\text { C } & -0.570576 & -2.925257 & -1.105059\end{array}$

$\begin{array}{llll}\mathrm{H} & 1.707973 & -2.082791 & -0.007052\end{array}$

$\mathrm{H} \quad-2.136204 \quad-1.395747 \quad-2.868171$

H $\quad-2.538343 \quad-1.096521 \quad-1.157628$

$\begin{array}{llll}\mathrm{H} & -1.663402 & 0.137569 & -2.083431\end{array}$

$\begin{array}{llll}\mathrm{H} & 0.406589 & -3.396392 & -1.248359\end{array}$

$\begin{array}{llll}\mathrm{H} & -0.880028 & -3.019114 & -0.055708\end{array}$

$\begin{array}{llll}\mathrm{H} & -1.303674 & -3.451763 & -1.724471\end{array}$

$\begin{array}{llll}\mathrm{H} & 4.877749 & 0.921338 & 1.070863\end{array}$

$\begin{array}{llll}\mathrm{H} & 5.304371 & -0.671305 & 1.753254\end{array}$

$\begin{array}{llll}\mathrm{H} & 5.497844 & -0.362942 & -0.001618\end{array}$

$\begin{array}{llll}\mathrm{H} & 0.512090 & 0.241391 & -1.653494\end{array}$

$\mathrm{H} \quad 4.154549 \quad 1.270276 \quad-1.078302$

$\begin{array}{llll}\mathrm{H} & 2.735847 & 0.836585 & -2.176245\end{array}$

$\begin{array}{llll}\mathrm{H} & -0.208223 & 2.876963 & 0.355118\end{array}$

$\begin{array}{llll}\mathrm{H} & 2.240386 & 3.201982 & 0.768392\end{array}$

$\begin{array}{llll}\mathrm{H} & 2.288088 & 1.582815 & 1.664452\end{array}$

$\begin{array}{llll}\mathrm{H} & 0.319265 & 0.255811 & 1.977230\end{array}$

$\begin{array}{llll}\mathrm{H} & -2.187811 & 1.814367 & -0.210949\end{array}$

$\mathrm{H} \quad-3.559116 \quad 3.636355 \quad-1.914362$

$\begin{array}{llll}\mathrm{H} & -4.327009 & 2.219780 & -1.166425\end{array}$

$\begin{array}{llll}\mathrm{H} & -3.759055 & 3.571231 & -0.150163\end{array}$

$\begin{array}{llll}\mathrm{H} & -2.239139 & -0.968323 & 1.359282\end{array}$

H $\quad-4.481928 \quad-1.147408 \quad 2.041837$
$\begin{array}{llll}\mathrm{H} & -4.191835 & -2.899256 & 2.093588\end{array}$

$\mathrm{H} \quad-3.371635 \quad-1.835722 \quad 3.255511$

Energies at $0 \mathrm{~K}$ and also $\mathrm{H}$ and $\mathrm{G} @ 298.15 \mathrm{~K}$.

$\mathrm{E}(0 \mathrm{~K}), \mathrm{E}+\mathrm{ZPE}(0 \mathrm{~K})$, Enthalpy, Free Energy

$-827.865163,-827.501373,-827.474294,-827.561943$

\section{DA TS4 - 2-MeOH catalyzed (bifurcated mode)} TS1

$\begin{array}{llll}\text { C } & 1.822046 & 1.918744 & 1.249795\end{array}$

$\begin{array}{llll}\text { C } & 0.453002 & 1.904441 & 1.030913\end{array}$

$\begin{array}{llll}\text { C } & -0.372260 & 0.833997 & 1.452879\end{array}$

$\begin{array}{llll}\mathrm{O} & -1.605102 & 0.732432 & 1.213828\end{array}$

$\begin{array}{llll}\text { C } & 2.870685 & 1.150240 & -0.536911\end{array}$

$\begin{array}{llll}\text { C } & 2.786317 & -0.226637 & -0.357551\end{array}$

$\begin{array}{llll}\text { O } & 3.777216 & -0.939588 & 0.257110\end{array}$

$\begin{array}{llll}\text { C } & 5.023851 & -0.313892 & 0.524486\end{array}$

$\begin{array}{llll}\text { C } & 1.660087 & -1.053445 & -0.618542\end{array}$

$\begin{array}{llll}\text { C } & 0.479272 & -0.631570 & -1.200295\end{array}$

$\begin{array}{llll}\mathrm{N} & -0.588159 & -1.399804 & -1.466756\end{array}$

C $\quad-1.843706 \quad-0.831626 \quad-1.957255$

$\begin{array}{llll}\text { C } & -0.622840 & -2.816125 & -1.115932\end{array}$

$\begin{array}{llll}\text { O } & -2.681060 & -1.804643 & 1.149405\end{array}$

$\begin{array}{llll}\text { C } & -3.701822 & -1.973787 & 2.111581\end{array}$

$\begin{array}{llll}\mathrm{O} & -2.081630 & 2.347757 & -0.993157\end{array}$

$\begin{array}{llll}\text { C } & -3.295486 & 3.074239 & -0.997542\end{array}$

H $\quad 1.772681 \quad-2.084044 \quad-0.302418$

H $\quad-2.073899 \quad-1.229376 \quad-2.954682$

$\mathrm{H} \quad-2.647812 \quad-1.104331 \quad-1.266170$

$\begin{array}{llll}\mathrm{H} & -1.780568 & 0.257845 & -1.997353\end{array}$

$\mathrm{H} \quad 0.343673 \quad-3.283791 \quad-1.321856$

$\mathrm{H} \quad-0.899215 \quad-2.947053 \quad-0.063698$

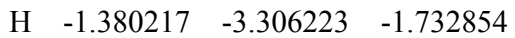

$\begin{array}{llll}\mathrm{H} & 4.921590 & 0.505908 & 1.246121\end{array}$

$\mathrm{H} \quad 5.658826 \quad-1.091824 \quad 0.952353$

$\begin{array}{llll}\mathrm{H} & 5.484875 & 0.070056 & -0.394221\end{array}$

$\mathrm{H} \quad 0.358956 \quad 0.404988 \quad-1.498814$

$\mathrm{H} \quad 3.827416 \quad 1.650903 \quad-0.448445$

$\begin{array}{llll}\mathrm{H} & 2.181814 & 1.620528 & -1.226081\end{array}$

$\begin{array}{llll}\mathrm{H} & -0.016577 & 2.689612 & 0.442976\end{array}$

$\begin{array}{llll}\mathrm{H} & 2.372701 & 2.848428 & 1.145864\end{array}$

$\begin{array}{llll}\mathrm{H} & 2.259417 & 1.205568 & 1.944190\end{array}$

$\begin{array}{llll}\mathrm{H} & 0.110819 & 0.044795 & 2.057868\end{array}$

$\begin{array}{llll}\mathrm{H} & -2.043123 & 1.810605 & -0.167163\end{array}$

$\mathrm{H} \quad-3.307616 \quad 3.695208 \quad-1.899434$

$\begin{array}{llll}\mathrm{H} & -4.182003 & 2.420319 & -1.023723\end{array}$

$\begin{array}{llll}\mathrm{H} & -3.390131 & 3.740088 & -0.125408\end{array}$

$\begin{array}{llll}\mathrm{H} & -2.315030 & -0.895248 & 1.249765\end{array}$

H $\quad-4.515309 \quad-1.239016 \quad 2.000622$

$\mathrm{H} \quad-4.129010 \quad-2.972848 \quad 1.973197$

H $\quad-3.325408-1.907616 \quad 3.145414$

Energies at $0 \mathrm{~K}$ and also H and G @ $298.15 \mathrm{~K}$ :

$\mathrm{E}(0 \mathrm{~K}), \mathrm{E}+\mathrm{ZPE}(0 \mathrm{~K})$, Enthalpy, Free Energy

$-827.860886,-827.495382,-827.470193,-827.551936$

DA 2-MeOH catalyzed (bifurcated mode) intermediate

$\begin{array}{llll}\text { C } & 5.184699 & -0.216271 & 0.521936\end{array}$

$\begin{array}{llll}\text { O } & 4.007269 & -0.896238 & 0.093667\end{array}$

$\begin{array}{llll}\text { C } & 2.900243 & -0.216758 & -0.297026\end{array}$

$\begin{array}{llll}\text { C } & 1.864038 & -1.029768 & -0.689178\end{array}$ 


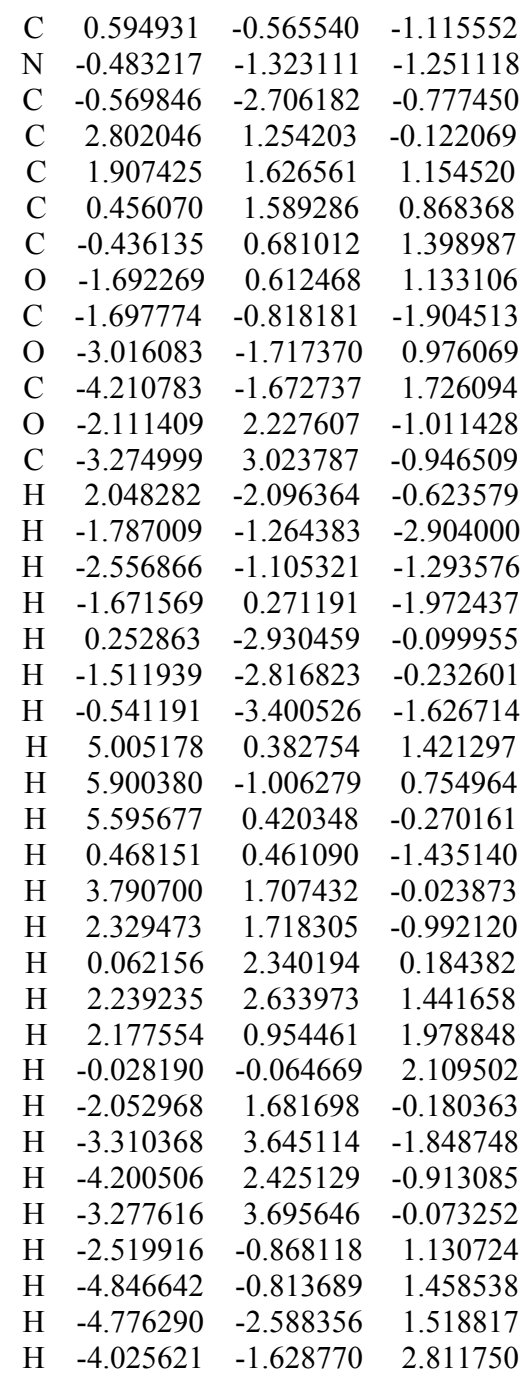

Energies at $0 \mathrm{~K}$ and also H and $\mathrm{G} @ 298.15 \mathrm{~K}$ : $\mathrm{E}(0 \mathrm{~K}), \mathrm{E}+\mathrm{ZPE}(0 \mathrm{~K})$, Enthalpy, Free Energy $-827.876713,-827.507877,-827.483336,-827.562916$

\begin{tabular}{crrr} 
DA 2-MeOH catalyzed (bifurcated mode) \\
\multicolumn{4}{c}{ TS2 } \\
C & -5.474882 & -0.007755 & -0.178378 \\
O & -4.260189 & -0.739565 & -0.278594 \\
C & -3.090842 & -0.180707 & 0.157433 \\
C & -2.905661 & 1.305322 & 0.242972 \\
C & -1.751235 & 1.747943 & -0.716097 \\
C & -0.402559 & 1.183623 & -0.355822 \\
C & 0.423404 & 0.611623 & -1.359519 \\
O & 1.645380 & 0.375229 & -1.224374 \\
C & -2.047073 & -1.014346 & 0.356754 \\
C & -0.740509 & -0.529303 & 0.808055 \\
N & 0.320477 & -1.381683 & 0.832622 \\
C & 1.415564 & -1.186509 & 1.777820 \\
C & 0.402768 & -2.522195 & -0.069422 \\
O & 2.705700 & 1.706047 & 0.985032 \\
C & 3.223924 & 2.954141 & 0.565287 \\
O & 3.522849 & -1.686595 & -0.966516 \\
C & 4.695282 & -0.976665 & -0.597323
\end{tabular}

$\begin{array}{rrrr}\mathrm{H} & -2.184267 & -2.075497 & 0.174889 \\ \mathrm{H} & 1.290594 & -1.817382 & 2.670894 \\ \mathrm{H} & 2.358362 & -1.441560 & 1.289075 \\ \mathrm{H} & 1.470373 & -0.138648 & 2.081012 \\ \mathrm{H} & -0.083874 & -2.284993 & -1.020829 \\ \mathrm{H} & 1.453160 & -2.746113 & -0.267855 \\ \mathrm{H} & -0.081744 & -3.416829 & 0.350898 \\ \mathrm{H} & -5.535198 & 0.798774 & -0.919968 \\ \mathrm{H} & -6.268960 & -0.729058 & -0.382419 \\ \mathrm{H} & -5.619978 & 0.410393 & 0.825610 \\ \mathrm{H} & -0.757416 & 0.126171 & 1.675718 \\ \mathrm{H} & -3.817972 & 1.847718 & -0.014956 \\ \mathrm{H} & -2.642224 & 1.604357 & 1.266351 \\ \mathrm{H} & 0.153658 & 1.717039 & 0.414367 \\ \mathrm{H} & -1.719383 & 2.845375 & -0.706569 \\ \mathrm{H} & -2.028695 & 1.450843 & -1.735727 \\ \mathrm{H} & -0.081123 & 0.260750 & -2.282615 \\ \mathrm{H} & 2.392124 & 1.223566 & 0.186457 \\ \mathrm{H} & 3.555301 & 3.493134 & 1.458937 \\ \mathrm{H} & 4.089662 & 2.846714 & -0.106673 \\ \mathrm{H} & 2.471229 & 3.575431 & 0.053533 \\ \mathrm{H} & 2.872946 & -1.025648 & -1.276837 \\ \mathrm{H} & 4.485497 & -0.175051 & 0.125618 \\ \mathrm{H} & 5.380337 & -1.693613 & -0.133196 \\ \mathrm{H} & 5.205261 & -0.536053 & -1.469000\end{array}$

Energies at $0 \mathrm{~K}$ and also $\mathrm{H}$ and $\mathrm{G} @ 298.15 \mathrm{~K}$ :

$\mathrm{E}(0 \mathrm{~K}), \mathrm{E}+\mathrm{ZPE}(0 \mathrm{~K})$, Enthalpy, Free Energy

$-827.871657,-827.503029,-827.478994,-827.557163$

\begin{tabular}{crrr} 
DA 2-MeOH catalyzed (bifurcated mode) \\
\multicolumn{4}{c}{ product complex } \\
C & -0.620146 & 0.396130 & 0.739636 \\
C & -0.128954 & -0.008456 & -0.727700 \\
C & -1.016261 & -1.100692 & -1.351033 \\
C & -2.465808 & -0.618403 & -1.484325 \\
C & -2.944300 & 0.059357 & -0.225388 \\
C & -2.126935 & 0.472332 & 0.752751 \\
C & 1.312813 & -0.407860 & -0.751563 \\
O & 2.247652 & 0.365843 & -0.941185 \\
O & -4.280271 & 0.375582 & -0.115120 \\
C & -5.240889 & -0.549391 & -0.606311 \\
N & -0.143001 & -0.352681 & 1.897431 \\
C & -0.516469 & -1.748593 & 2.021272 \\
C & 1.227217 & -0.097789 & 2.315881 \\
O & 4.060164 & -1.633555 & -0.077338 \\
C & 5.005381 & -2.158419 & -0.991094 \\
O & 1.382223 & 2.935023 & -0.032900 \\
C & 2.136798 & 4.113638 & -0.247129 \\
H & -2.566575 & 0.943837 & 1.627824 \\
H & 1.328949 & -0.323305 & 3.385430 \\
H & 1.980871 & -0.709630 & 1.785829 \\
H & 1.473347 & 0.956891 & 2.158957 \\
H & -1.569133 & -1.878164 & 1.754632 \\
H & 0.092320 & -2.432703 & 1.396629 \\
H & -0.386150 & -2.066974 & 3.063150 \\
H & -5.103271 & -1.549535 & -0.172423 \\
H & -6.213114 & -0.160317 & -0.295741 \\
H & -5.228501 & -0.626606 & -1.701241 \\
H & -0.229890 & 1.410659 & 0.877676 \\
H & -3.104304 & -1.474249 & -1.734456 \\
H & -2.559314 & 0.082982 & -2.327726
\end{tabular}




$\begin{array}{rrrr}\mathrm{H} & -0.215090 & 0.915431 & -1.314123 \\ \mathrm{H} & -0.638600 & -1.386108 & -2.341054 \\ \mathrm{H} & -0.984954 & -2.003452 & -0.730576 \\ \mathrm{H} & 1.544879 & -1.476015 & -0.581488 \\ \mathrm{H} & 1.831616 & 2.188486 & -0.471224 \\ \mathrm{H} & 1.612055 & 4.931744 & 0.255301 \\ \mathrm{H} & 3.151574 & 4.048801 & 0.175786 \\ \mathrm{H} & 2.224905 & 4.370562 & -1.314677 \\ \mathrm{H} & 3.737347 & -0.781583 & -0.424942 \\ \mathrm{H} & 5.902682 & -1.525920 & -1.081245 \\ \mathrm{H} & 5.318565 & -3.135123 & -0.609629 \\ \mathrm{H} & 4.587970 & -2.305210 & -2.000329\end{array}$

Energies at 0K and also H and G @ 298.15 K: $\mathrm{E}(0 \mathrm{~K}), \mathrm{E}+\mathrm{ZPE}(0 \mathrm{~K})$, Enthalpy, Free Energy $-827.889031,-827.519533,-827.494965,-827.577686$

$$
\begin{array}{rrrr}
\mathrm{H} & 5.341772 & -0.436345 & -1.827655 \\
\mathrm{H} & 5.384948 & 0.459241 & 0.549920 \\
\mathrm{H} & 5.394117 & -1.294336 & 0.402250
\end{array}
$$

Energies at 0K and also H and G @ 298.15 K:

\begin{tabular}{|c|c|c|c|}
\hline \multicolumn{4}{|c|}{ TS5 - DA 1,4-butanediol } \\
\hline & & & \\
\hline & 1.560786 & -2.079 & 14737 \\
\hline & & & \\
\hline & & & \\
\hline J & & & \\
\hline & -2.4 & -1.5 & \\
\hline & & & \\
\hline 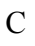 & & & \\
\hline $\mathrm{C}$ & & & \\
\hline $\mathrm{I}$ & & & \\
\hline C & & & \\
\hline 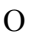 & & & \\
\hline & & -1.3 & \\
\hline t & & & \\
\hline ) & & & \\
\hline & & & \\
\hline & & & \\
\hline & & & \\
\hline & & & \\
\hline 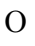 & & & \\
\hline & & & \\
\hline & & & \\
\hline & & & \\
\hline 1 & & & \\
\hline & & & \\
\hline & & & \\
\hline I & & & \\
\hline & & & \\
\hline & & -0. & \\
\hline & & & \\
\hline & & & \\
\hline & & & \\
\hline & & -1 & \\
\hline $\mathrm{H}$ & & & \\
\hline 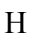 & & & \\
\hline 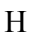 & & & \\
\hline 1 & & & \\
\hline $\mathrm{H}$ & & & \\
\hline $\mathrm{H}$ & & -0. & \\
\hline 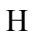 & & & \\
\hline & & -0.2 & \\
\hline & & & \\
\hline & & & \\
\hline & & & \\
\hline & & -1.0 & \\
\hline & & & \\
\hline & 5.081386 & -1.732697 & 0.562776 \\
\hline
\end{tabular}

$\mathrm{E}(0 \mathrm{~K}), \mathrm{E}+\mathrm{ZPE}(0 \mathrm{~K})$, Enthalpy, Free Energy -905.296359, -904.893260, -904.867178, -904.950778

Energies at $0 \mathrm{~K}$ and also H and G @ 298.15 K:

$\mathrm{E}(0 \mathrm{~K}), \mathrm{E}+\mathrm{ZPE}(0 \mathrm{~K})$, Enthalpy, Free Energy -905.287921, -904.883244, -904.858968, -904.937018 


\begin{tabular}{|c|c|c|}
\hline \multicolumn{3}{|c|}{$\begin{array}{c}\text { DA 1,4-butanediol catalyzed } \\
\text { intermediate }\end{array}$} \\
\hline 3.610717 & -3.101304 & -0.137829 \\
\hline 2.817874 & -2.134652 & -0.818064 \\
\hline 2.765478 & -0.846544 & -0.396869 \\
\hline 3.409563 & -0.413981 & 0.871322 \\
\hline 2.322795 & -0.218565 & 2.035211 \\
\hline 1.451646 & 0.970784 & 1.873812 \\
\hline 0.105153 & 0.892112 & 1.543317 \\
\hline-0.692788 & 1.869729 & 1.364953 \\
\hline 1.921798 & -0.042332 & -1.120627 \\
\hline 1.731972 & 1.324651 & -0.798513 \\
\hline 0.775689 & 2.117850 & -1.270542 \\
\hline 0.758498 & 3.537967 & -0.915028 \\
\hline-0.388947 & 1.6 & 487 \\
\hline-2.805766 & & \\
\hline-3.676773 & 0.204092 & 0073 \\
\hline-4.487392 & -0.8 & 9161 \\
\hline-3.724095 & -2.159001 & 463 \\
\hline-2.767478 & -2.0 & -1.1 \\
\hline-1.600948 & -1.2 & -0 . \\
\hline 1.326667 & -0.5 & 667 \\
\hline 0.475889 & 4.129557 & -1.7 \\
\hline 0.046031 & 3.6959 & -0.1 \\
\hline 1.754430 & 3.8 & -0.5 \\
\hline-0.288035 & 1.9 & 7099 \\
\hline-0.509962 & 0.56 & -1.910727 \\
\hline-1.287961 & 2.111599 & -1.612819 \\
\hline 3.285874 & -3.239313 & 9501 \\
\hline 3.458713 & -4.03 & -0.685901 \\
\hline 4.676286 & -2.8 & -0.158311 \\
\hline 2.500007 & 1.843563 & -0.242090 \\
\hline 4.168113 & -1.125903 & 1.203592 \\
\hline 3.919974 & 0.545477 & 836 \\
\hline 1.868567 & 1.9 & 2.098273 \\
\hline 2.912880 & -0.167588 & 2.960156 \\
\hline 1.725658 & -1.137243 & 2.086960 \\
\hline-0.285457 & -0.132853 & 1.378503 \\
\hline-3.108176 & -0.233853 & 1.996002 \\
\hline-4.373637 & 0.938663 & 1.593844 \\
\hline-4.982959 & -0.441057 & -0.414538 \\
\hline-5.289064 & -1.188041 & 1.148780 \\
\hline-4.467258 & -2.926060 & -0.233312 \\
\hline-3.154968 & -2.554689 & 0.882937 \\
\hline-3.319909 & -1.588870 & -2.019361 \\
\hline-2.427899 & -3.014374 & -1.469160 \\
\hline-1.931287 & -0.410871 & -0.500838 \\
\hline-2.066915 & 1.324871 & 0.750968 \\
\hline
\end{tabular}

Energies at $0 \mathrm{~K}$ and also H and G @ 298.15 K: $\mathrm{E}(0 \mathrm{~K}), \mathrm{E}+\mathrm{ZPE}(0 \mathrm{~K})$, Enthalpy, Free Energy $-905.302342,-904.895536,-904.871484,-904.949837$

\begin{tabular}{lrrr}
\multicolumn{4}{c}{ DA 1,4-butanediol catalyzed } \\
C & 0.708257 & 2.130139 & -1.519393 \\
N & -0.573084 & 1.959788 & -0.844720 \\
C & -1.158796 & 3.159039 & -0.264660 \\
C & -1.171306 & 0.750119 & -0.750515 \\
C & -2.612399 & 0.639966 & -0.624862 \\
C & -3.229360 & -0.532508 & -0.336410 \\
O & -4.590918 & -0.491564 & -0.227411
\end{tabular}

$\begin{array}{lrrr}\mathrm{C} & -5.340059 & -1.699005 & -0.191400 \\ \mathrm{C} & -2.438966 & -1.745569 & 0.035327 \\ \mathrm{C} & -1.549171 & -1.434848 & 1.297427 \\ \mathrm{C} & -0.473504 & -0.424727 & 1.047163 \\ \mathrm{C} & -0.314168 & 0.702566 & 1.881513 \\ \mathrm{O} & 0.644188 & 1.513198 & 1.875225 \\ \mathrm{O} & 2.896287 & 0.746043 & 0.617948 \\ \mathrm{C} & 3.495611 & -0.347209 & 1.306998 \\ \mathrm{C} & 4.696111 & -0.864459 & 0.510355 \\ \mathrm{C} & 4.450108 & -1.099944 & -0.997948 \\ \mathrm{C} & 3.145540 & -1.818845 & -1.366524 \\ \mathrm{O} & 2.002756 & -0.968341 & -1.299370 \\ \mathrm{H} & -3.224861 & 1.528611 & -0.743945 \\ \mathrm{H} & 0.600403 & 2.836637 & -2.354404 \\ \mathrm{H} & 1.463951 & 2.513364 & -0.826579 \\ \mathrm{H} & 1.053835 & 1.173284 & -1.913676 \\ \mathrm{H} & -1.735771 & 3.729758 & -1.007771 \\ \mathrm{H} & -1.816153 & 2.893923 & 0.565739 \\ \mathrm{H} & -0.361066 & 3.796224 & 0.125215 \\ \mathrm{H} & -5.209674 & -2.238583 & 0.754901 \\ \mathrm{H} & -6.385389 & -1.396214 & -0.280953 \\ \mathrm{H} & -5.086230 & -2.363516 & -1.026689 \\ \mathrm{H} & -0.645094 & -0.027386 & -1.294081 \\ \mathrm{H} & -3.077824 & -2.606691 & 0.242936 \\ \mathrm{H} & -1.769974 & -2.038736 & -0.783061 \\ \mathrm{H} & 0.408171 & -0.782520 & 0.520642 \\ \mathrm{H} & -1.114727 & -2.392766 & 1.613815 \\ \mathrm{H} & -2.212398 & -1.105247 & 2.107795 \\ \mathrm{H} & -1.175194 & 0.933247 & 2.545205 \\ \mathrm{H} & 2.091857 & 1.048848 & 1.129234 \\ \mathrm{H} & 3.827043 & -0.037725 & 2.309106 \\ \mathrm{H} & 2.763862 & -1.159257 & 1.446917 \\ \mathrm{H} & 2.229712 & -0.241039 & -0.671464 \\ \mathrm{H} & 3.005653 & -2.700701 & -0.717519 \\ \mathrm{H} & 3.211628 & -2.189744 & -2.397626 \\ \mathrm{H} & 4.457639 & -0.138643 & -1.526182 \\ \mathrm{H} & 5.295899 & -1.677779 & -1.394282 \\ \mathrm{H} & 5.528420 & -0.156874 & 0.613814 \\ \mathrm{H} & 5.021978 & -1.801967 & 0.982382\end{array}$

Energies at $0 \mathrm{~K}$ and also $\mathrm{H}$ and $\mathrm{G} @ 298.15 \mathrm{~K}$ : $\mathrm{E}(0 \mathrm{~K}), \mathrm{E}+\mathrm{ZPE}(0 \mathrm{~K})$, Enthalpy, Free Energy $-905.300087,-904.893011,-904.869665,-904.945671$

\begin{tabular}{lrrr}
\multicolumn{4}{c}{ DA 1,4-butanediol catalyzed } \\
product complex \\
C & 2.283192 & -0.197339 & -0.782282 \\
C & 2.061400 & 1.164449 & -0.169168 \\
C & 1.231391 & 1.061852 & 1.189817 \\
C & 1.637511 & -0.197582 & 2.021866 \\
C & 2.866930 & -0.963602 & 1.478196 \\
C & 2.700874 & -1.201066 & -0.003828 \\
N & 1.625385 & 2.144253 & -1.156508 \\
C & 1.757620 & 3.527537 & -0.728635 \\
C & -0.253528 & 0.980794 & 0.967464 \\
O & -1.053936 & 1.768369 & 1.458818 \\
O & 2.880312 & -2.464982 & -0.516829 \\
C & 4.159886 & -3.048424 & -0.297465 \\
C & 0.362101 & 1.905689 & -1.847112 \\
O & -3.469751 & 1.009906 & 0.305977 \\
C & -4.054014 & 0.027917 & 1.165450 \\
C & -4.646380 & -1.129124 & 0.360466
\end{tabular}




$\begin{array}{rrrr}\mathrm{C} & -3.647292 & -2.110420 & -0.287944 \\ \mathrm{C} & -2.954533 & -1.634491 & -1.571270 \\ \mathrm{O} & -2.043654 & -0.565360 & -1.380949 \\ \mathrm{H} & 2.160175 & -0.331958 & -1.851171 \\ \mathrm{H} & 1.643501 & 4.185447 & -1.598228 \\ \mathrm{H} & 1.008890 & 3.839661 & 0.023281 \\ \mathrm{H} & 2.756277 & 3.695158 & -0.308464 \\ \mathrm{H} & 0.367604 & 2.452755 & -2.798259 \\ \mathrm{H} & 0.223907 & 0.844775 & -2.066111 \\ \mathrm{H} & -0.522539 & 2.246771 & -1.282016 \\ \mathrm{H} & 4.394549 & -3.150964 & 0.770786 \\ \mathrm{H} & 4.124448 & -4.043310 & -0.746840 \\ \mathrm{H} & 4.951725 & -2.459626 & -0.780852 \\ \mathrm{H} & 3.039920 & 1.535564 & 0.174518 \\ \mathrm{H} & 2.960913 & -1.912307 & 2.016641 \\ \mathrm{H} & 3.781503 & -0.387083 & 1.681766 \\ \mathrm{H} & 1.417169 & 1.971006 & 1.770203 \\ \mathrm{H} & 1.806046 & 0.097326 & 3.062947 \\ \mathrm{H} & 0.801846 & -0.909378 & 2.037063 \\ \mathrm{H} & -0.610888 & 0.136784 & 0.347350 \\ \mathrm{H} & -3.304864 & -0.357170 & 1.873768 \\ \mathrm{H} & -4.853109 & 0.497921 & 1.757977 \\ \mathrm{H} & -5.315323 & -0.712695 & -0.406183 \\ \mathrm{H} & -5.286888 & -1.694885 & 1.050930 \\ \mathrm{H} & -4.190820 & -3.033423 & -0.535731 \\ \mathrm{H} & -2.877325 & -2.390952 & 0.445121 \\ \mathrm{H} & -3.723459 & -1.366306 & -2.317496 \\ \mathrm{H} & -2.373469 & -2.462559 & -1.993259 \\ \mathrm{H} & -2.553410 & 0.151984 & -0.939175 \\ \mathrm{H} & -2.738791 & 1.447075 & 0.797533\end{array}$

Energies at $0 \mathrm{~K}$ and also H and G@298.15 K: $\mathrm{E}(0 \mathrm{~K}), \mathrm{E}+\mathrm{ZPE}(0 \mathrm{~K})$, Enthalpy, Free Energy $-905.317826,-904.909095,-904.885541,-904.963504$

$\begin{array}{crrr}\mathrm{C} & -4.665408 & -1.663273 & -0.526294 \\ \mathrm{H} & -2.539710 & -1.996987 & -0.346989 \\ \mathrm{C} & -5.696002 & -0.726566 & -0.419001 \\ \mathrm{H} & -6.224089 & 1.318040 & 0.028988 \\ \mathrm{H} & -4.886914 & -2.687873 & -0.810890 \\ \mathrm{H} & -6.721103 & -1.025760 & -0.621201 \\ \mathrm{O} & 3.003090 & -1.946824 & -0.495980 \\ \mathrm{Si} & 3.129279 & -3.581123 & -0.177172 \\ \mathrm{H} & 1.806475 & -4.266914 & -0.178971 \\ \mathrm{H} & 3.952877 & -4.126634 & -1.282168 \\ \mathrm{H} & 3.789375 & -3.834021 & 1.131530\end{array}$

Energies at $0 \mathrm{~K}$ and also $\mathrm{H}$ and $\mathrm{G} @ 298.15 \mathrm{~K}$ : $\mathrm{E}(0 \mathrm{~K}), \mathrm{E}+\mathrm{ZPE}(0 \mathrm{~K})$, Enthalpy, Free Energy -1001.491457, -1001.200541, -1001.179155, $-1001.255774$

\begin{tabular}{lrrr} 
& \multicolumn{3}{c}{ TS6 - hDA uncatalyzed } \\
& & TS & \\
$\mathrm{C}$ & 2.489208 & 0.476198 & -0.250405 \\
$\mathrm{C}$ & 1.500602 & 1.164603 & 0.456281 \\
$\mathrm{C}$ & 0.291104 & 1.494190 & -0.145930 \\
$\mathrm{C}$ & -0.058287 & 1.085971 & -1.474727 \\
$\mathrm{~N}$ & 3.580108 & -0.095291 & 0.289809 \\
$\mathrm{H}$ & 2.482214 & 0.479383 & -1.328705 \\
$\mathrm{H}$ & 1.578096 & 1.321817 & 1.526179 \\
$\mathrm{H}$ & -1.028587 & 1.432263 & -1.831636 \\
$\mathrm{H}$ & 0.715416 & 1.256265 & -2.221725 \\
$\mathrm{C}$ & -0.053980 & -0.759529 & -1.514902 \\
$\mathrm{O}$ & 1.125120 & -1.207221 & -1.237290 \\
$\mathrm{H}$ & -0.363174 & -0.806344 & -2.583103 \\
$\mathrm{C}$ & 4.409216 & -0.959099 & -0.544676 \\
$\mathrm{C}$ & 3.679101 & -0.305472 & 1.729212 \\
$\mathrm{H}$ & 5.465514 & -0.829691 & -0.285272 \\
$\mathrm{H}$ & 4.130919 & -2.012398 & -0.413663 \\
$\mathrm{H}$ & 4.265020 & -0.700313 & -1.594580 \\
$\mathrm{H}$ & 4.678801 & -0.678265 & 1.963622 \\
$\mathrm{H}$ & 3.533894 & 0.635735 & 2.266399 \\
$\mathrm{H}$ & 2.935202 & -1.033670 & 2.080117 \\
$\mathrm{C}$ & -1.210183 & -1.193322 & -0.623303 \\
$\mathrm{C}$ & -0.944288 & -1.752904 & 0.630506 \\
$\mathrm{C}$ & -2.541481 & -1.078933 & -1.042311 \\
$\mathrm{C}$ & -1.987590 & -2.165298 & 1.460406 \\
$\mathrm{H}$ & 0.094411 & -1.874396 & 0.920713 \\
$\mathrm{C}$ & -3.588184 & -1.495326 & -0.218812 \\
$\mathrm{H}$ & -2.759377 & -0.668247 & -2.027818 \\
$\mathrm{C}$ & -3.313489 & -2.035608 & 1.040397 \\
$\mathrm{H}$ & -1.767394 & -2.598984 & 2.433413 \\
$\mathrm{H}$ & -4.616782 & -1.405235 & -0.560418 \\
$\mathrm{H}$ & -4.126691 & -2.363403 & 1.683297 \\
$\mathrm{O}$ & -0.640303 & 2.020598 & 0.694358 \\
$\mathrm{Si}$ & -2.057304 & 2.845887 & 0.323740 \\
$\mathrm{H}$ & -3.086997 & 1.977875 & -0.294555 \\
$\mathrm{H}$ & -2.535613 & 3.350202 & 1.630430 \\
$\mathrm{H}$ & -1.762404 & 3.982176 & -0.590674
\end{tabular}

Energies at 0K and also H and G @ $298.15 \mathrm{~K}$ : $\mathrm{E}(0 \mathrm{~K}), \mathrm{E}+\mathrm{ZPE}(0 \mathrm{~K})$, Enthalpy, Free Energy $-1001.460442,-1001.168010,-1001.148616$, $-1001.216807$ 


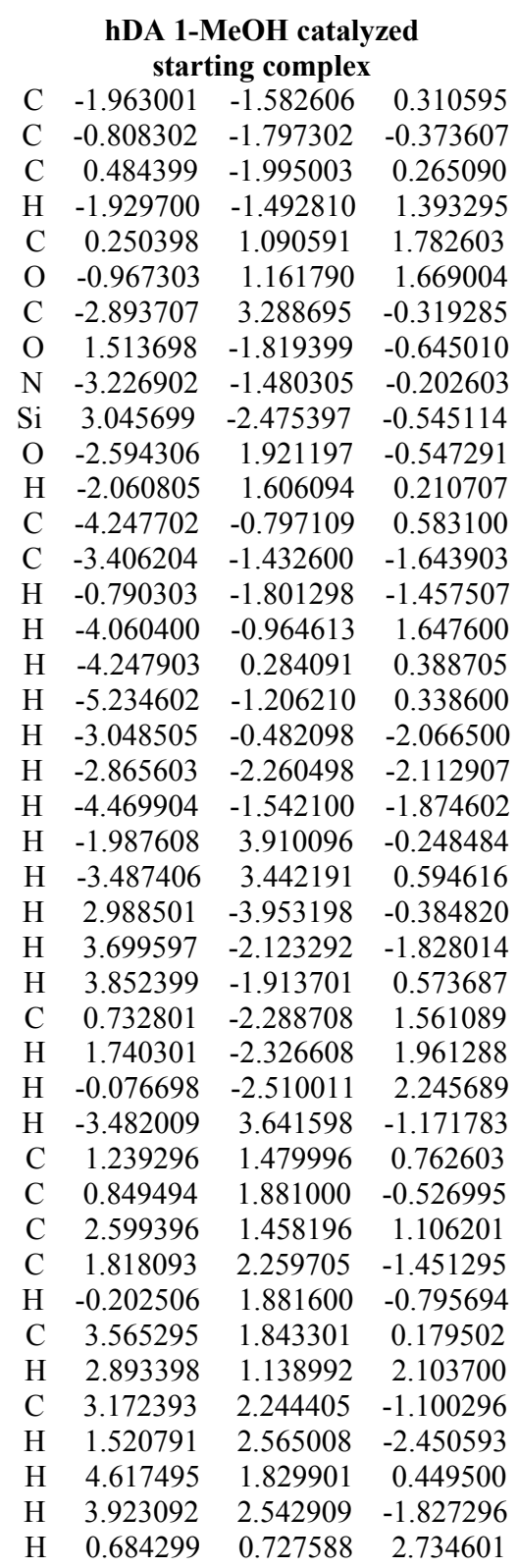

Energies at $0 \mathrm{~K}$ and also H and G @ 298.15 K: $\mathrm{E}(0 \mathrm{~K}), \mathrm{E}+\mathrm{ZPE}(0 \mathrm{~K})$, Enthalpy, Free Energy -1117.219744, -1116.874570, -1116.848914, $-1116.933492$

\section{hDA 1-MeOH catalyzed}

TS

$\begin{array}{rrrr}\mathrm{C} & -2.130327 & 0.110948 & -1.164317 \\ \mathrm{C} & -1.847507 & 1.358618 & -0.623832 \\ \mathrm{C} & -0.534680 & 1.829736 & -0.490480 \\ \mathrm{H} & -1.342022 & -0.454615 & -1.642530 \\ \mathrm{C} & 0.770912 & -0.349834 & 0.441413 \\ \mathrm{O} & -0.204940 & -1.158715 & 0.242938 \\ \mathrm{C} & -2.191761 & -2.401725 & 2.732768 \\ \mathrm{O} & -0.444430 & 2.997414 & 0.204919 \\ \mathrm{~N} & -3.320485 & -0.499399 & -1.159155 \\ \mathrm{Si} & 0.893169 & 3.977565 & 0.484980\end{array}$

$\begin{array}{lrrr}\mathrm{O} & -2.495259 & -1.591411 & 1.617781 \\ \mathrm{H} & -1.637688 & -1.348533 & 1.181652 \\ \mathrm{C} & -3.396517 & -1.920642 & -1.493768 \\ \mathrm{C} & -4.430978 & 0.026844 & -0.366724 \\ \mathrm{H} & -2.623344 & 1.956800 & -0.161251 \\ \mathrm{H} & -2.549895 & -2.193355 & -2.127046 \\ \mathrm{H} & -3.357652 & -2.510653 & -0.570941 \\ \mathrm{H} & -4.325938 & -2.125804 & -2.034760 \\ \mathrm{H} & -4.226430 & -0.121694 & 0.699601 \\ \mathrm{H} & -4.581864 & 1.087622 & -0.582876 \\ \mathrm{H} & -5.341551 & -0.510184 & -0.641934 \\ \mathrm{H} & -3.136013 & -2.749995 & 3.166881 \\ \mathrm{H} & -1.643484 & -1.855034 & 3.518211 \\ \mathrm{H} & 1.546678 & 4.362113 & -0.792870 \\ \mathrm{H} & 0.336131 & 5.168970 & 1.162192 \\ \mathrm{H} & 1.888291 & 3.312769 & 1.364114 \\ \mathrm{H} & 0.765102 & 0.263201 & 1.365533 \\ \mathrm{C} & 0.638845 & 1.107711 & -0.830926 \\ \mathrm{H} & 1.576701 & 1.657980 & -0.782086 \\ \mathrm{H} & 0.583167 & 0.487462 & -1.720619 \\ \mathrm{C} & 4.751998 & -1.753447 & -0.495172 \\ \mathrm{C} & 4.582293 & -0.639593 & 0.331000 \\ \mathrm{C} & 3.298931 & -0.181771 & 0.629547 \\ \mathrm{C} & 2.171375 & -0.828327 & 0.108948 \\ \mathrm{C} & 2.348553 & -1.950739 & -0.707373 \\ \mathrm{C} & 3.630614 & -2.408076 & -1.011221 \\ \mathrm{H} & 5.750962 & -2.113332 & -0.727779 \\ \mathrm{H} & 5.449423 & -0.131801 & 0.746293 \\ \mathrm{H} & 3.168472 & 0.682937 & 1.278762 \\ \mathrm{H} & 1.464194 & -2.461286 & -1.076040 \\ \mathrm{H} & 3.757008 & -3.283071 & -1.644476 \\ \mathrm{H} & -1.594701 & -3.287133 & 2.462066\end{array}$

Energies at 0K and also H and G @ 298.15 K: $\mathrm{E}(0 \mathrm{~K}), \mathrm{E}+\mathrm{ZPE}(0 \mathrm{~K})$, Enthalpy, Free Energy -1117.201391, -1116.854971, -1116.831204, $-1116.909754$

hDA 2-MeOH catalyzed (cooperative mode)

\begin{tabular}{lrrr}
\multicolumn{4}{c}{ starting complex } \\
$\mathrm{C}$ & -2.487998 & 1.172506 & -0.541899 \\
$\mathrm{C}$ & -1.667396 & 2.175204 & -0.135999 \\
$\mathrm{C}$ & -0.239696 & 2.205702 & -0.422300 \\
$\mathrm{H}$ & -2.070300 & 0.298805 & -1.037199 \\
$\mathrm{C}$ & 1.021898 & -1.300401 & 0.407001 \\
$\mathrm{O}$ & 0.541398 & -1.220999 & 1.535301 \\
$\mathrm{C}$ & -1.950407 & -3.477696 & -1.875498 \\
$\mathrm{C}$ & -2.197405 & -3.098394 & 2.299502 \\
$\mathrm{O}$ & 0.436006 & 2.972900 & 0.512700 \\
$\mathrm{~N}$ & -3.853698 & 1.127808 & -0.421699 \\
$\mathrm{Si}$ & 1.965207 & 3.622198 & 0.353799 \\
$\mathrm{O}$ & -1.807504 & -2.178096 & -1.331499 \\
$\mathrm{H}$ & -1.997804 & -2.217095 & -0.364799 \\
$\mathrm{O}$ & -2.105803 & -1.995495 & 1.407301 \\
$\mathrm{H}$ & -1.232703 & -1.562396 & 1.556001 \\
$\mathrm{C}$ & -4.512100 & -0.171591 & -0.413598 \\
$\mathrm{C}$ & -4.545396 & 2.204310 & 0.258301 \\
$\mathrm{H}$ & -2.038894 & 3.002005 & 0.460600 \\
$\mathrm{H}$ & -5.520900 & -0.076689 & -0.832298 \\
$\mathrm{H}$ & -3.935002 & -0.870792 & -1.022998 \\
$\mathrm{H}$ & -4.588101 & -0.591590 & 0.601102 \\
$\mathrm{H}$ & -4.371196 & 2.200209 & 1.347201
\end{tabular}




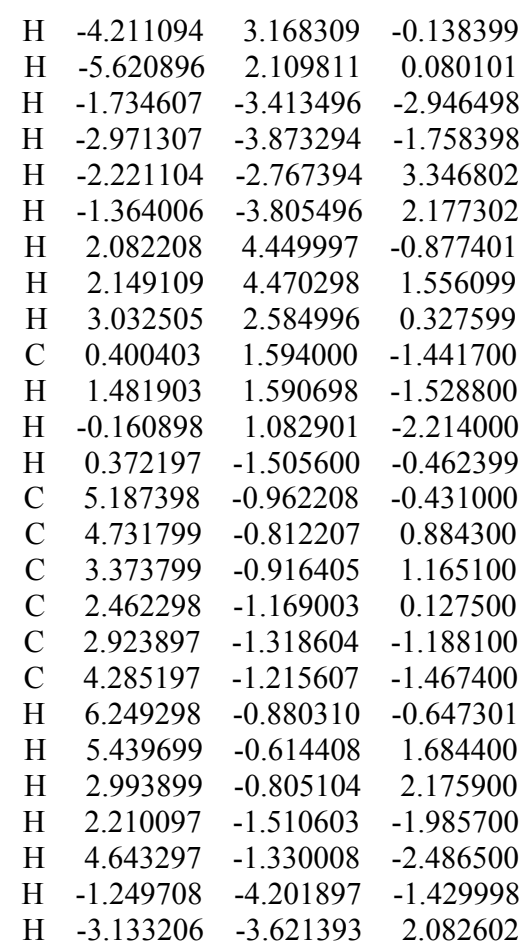

Energies at $0 \mathrm{~K}$ and also H and G @ 298.15 K: $\mathrm{E}(0 \mathrm{~K}), \mathrm{E}+\mathrm{ZPE}(0 \mathrm{~K})$, Enthalpy, Free Energy -1232.952094, -1232.553023, -1232.522798, $-1232.620916$

TS7 - hDA 2-MeOH catalyzed (cooperative mode) TS

$\begin{array}{rrrr}\mathrm{C} & -1.710743 & -1.479284 & -0.324611 \\ \mathrm{C} & -0.441719 & -2.025315 & -0.193285 \\ \mathrm{C} & 0.507998 & -1.478175 & 0.684437 \\ \mathrm{H} & -2.058215 & -0.745145 & 0.390947 \\ \mathrm{C} & 0.410879 & 1.166910 & 0.048359 \\ \mathrm{O} & -0.470231 & 0.940998 & -0.857622 \\ \mathrm{C} & -4.012683 & 1.062454 & 2.740420 \\ \mathrm{C} & -2.628711 & 3.532390 & -0.610598 \\ \mathrm{O} & 1.709214 & -2.113711 & 0.650928 \\ \mathrm{~N} & -2.626670 & -1.831622 & -1.232569 \\ \mathrm{Si} & 2.981219 & -2.057499 & 1.750509 \\ \mathrm{O} & -3.216145 & 0.580746 & 1.675770 \\ \mathrm{H} & -3.115429 & 1.291765 & 0.992051 \\ \mathrm{O} & -2.769696 & 2.130748 & -0.470152 \\ \mathrm{H} & -1.880429 & 1.695055 & -0.669123 \\ \mathrm{C} & -3.958182 & -1.230100 & -1.218047 \\ \mathrm{C} & -2.313577 & -2.765233 & -2.306004 \\ \mathrm{H} & -0.116610 & -2.859028 & -0.805772 \\ \mathrm{H} & -4.726092 & -2.012484 & -1.179403 \\ \mathrm{H} & -4.054270 & -0.588917 & -0.340517 \\ \mathrm{H} & -4.114249 & -0.624967 & -2.119111 \\ \mathrm{H} & -1.352220 & -2.508088 & -2.762704 \\ \mathrm{H} & -2.264197 & -3.798712 & -1.938617 \\ \mathrm{H} & -3.091191 & -2.704304 & -3.070143 \\ \mathrm{H} & -4.136802 & 0.247670 & 3.461118 \\ \mathrm{H} & -5.013920 & 1.377389 & 2.406964 \\ \mathrm{H} & -2.292381 & 3.804208 & -1.621371 \\ \mathrm{H} & -1.918066 & 3.962958 & 0.112450\end{array}$

$\begin{array}{rrrr}\mathrm{H} & 2.489179 & -2.407062 & 3.110306 \\ \mathrm{H} & 3.922048 & -3.092908 & 1.266479 \\ \mathrm{H} & 3.651215 & -0.737792 & 1.795761 \\ \mathrm{C} & 0.344989 & -0.264694 & 1.395673 \\ \mathrm{H} & 1.112008 & -0.019409 & 2.128667 \\ \mathrm{H} & -0.660378 & -0.038588 & 1.741237 \\ \mathrm{H} & 0.183747 & 1.932555 & 0.816906 \\ \mathrm{C} & 4.555677 & 1.319677 & -1.148469 \\ \mathrm{C} & 3.621709 & 0.625864 & -1.923337 \\ \mathrm{C} & 2.283024 & 0.576946 & -1.534621 \\ \mathrm{C} & 1.863550 & 1.207879 & -0.357059 \\ \mathrm{C} & 2.803463 & 1.907393 & 0.410064 \\ \mathrm{C} & 4.141245 & 1.966500 & 0.018349 \\ \mathrm{H} & 5.597004 & 1.364498 & -1.457214 \\ \mathrm{H} & 3.937463 & 0.131306 & -2.838921 \\ \mathrm{H} & 1.538477 & 0.066910 & -2.137578 \\ \mathrm{H} & 2.480472 & 2.416131 & 1.317251 \\ \mathrm{H} & 4.858638 & 2.520376 & 0.619039 \\ \mathrm{H} & -3.544776 & 1.910625 & 3.264661 \\ \mathrm{H} & -3.608068 & 3.992773 & -0.441514\end{array}$

Energies at 0K and also H and G @ 298.15 K: $\mathrm{E}(0 \mathrm{~K}), \mathrm{E}+\mathrm{ZPE}(0 \mathrm{~K})$, Enthalpy, Free Energy -1232.937861, -1232.538010, -1232.509578, $-1232.601045$

hDA 2-MeOH catalyzed (bifurcated mode)

\begin{tabular}{rrrr}
\multicolumn{3}{c}{ starting complex } \\
C & 2.752020 & 1.202873 & 1.408542 \\
$\mathrm{C}$ & 3.855635 & 1.735687 & 0.748611 \\
$\mathrm{C}$ & 3.677670 & 2.406476 & -0.465012 \\
$\mathrm{C}$ & 2.398982 & 2.544102 & -1.014913 \\
$\mathrm{C}$ & 1.291250 & 2.018595 & -0.356391 \\
$\mathrm{C}$ & 1.464812 & 1.344196 & 0.865224 \\
$\mathrm{C}$ & 0.324454 & 0.793182 & 1.606784 \\
$\mathrm{O}$ & -0.862088 & 0.956750 & 1.308532 \\
$\mathrm{O}$ & -2.639874 & -1.041014 & 2.265511 \\
$\mathrm{C}$ & -3.130822 & -0.870871 & 3.582838 \\
$\mathrm{O}$ & -2.092531 & 2.240238 & -0.948964 \\
$\mathrm{C}$ & -2.409965 & 3.525656 & -0.440277 \\
$\mathrm{C}$ & 0.590379 & -2.182151 & 1.007278 \\
$\mathrm{C}$ & 0.685021 & -1.877051 & -0.309989 \\
$\mathrm{C}$ & -0.367222 & -1.436735 & -1.209084 \\
$\mathrm{C}$ & -1.686986 & -1.390086 & -0.873100 \\
$\mathrm{~N}$ & -2.728267 & -1.027321 & -1.675976 \\
$\mathrm{C}$ & -3.986512 & -0.617846 & -1.062513 \\
$\mathrm{O}$ & 1.900560 & -1.916775 & -0.971852 \\
$\mathrm{Si}$ & 3.308966 & -2.655867 & -0.470785 \\
$\mathrm{C}$ & -2.470770 & -0.506251 & -3.007459 \\
$\mathrm{H}$ & -2.000813 & -1.689423 & 0.122330 \\
$\mathrm{H}$ & -1.713186 & 1.718045 & -0.214850 \\
$\mathrm{H}$ & -2.098065 & -0.261514 & 2.027722 \\
$\mathrm{H}$ & -0.025915 & -1.137295 & -2.193488 \\
$\mathrm{H}$ & -4.832531 & -0.986480 & -1.655341 \\
$\mathrm{H}$ & -4.050176 & -1.028554 & -0.052103 \\
$\mathrm{H}$ & -4.047274 & 0.477359 & -0.996132 \\
$\mathrm{H}$ & -1.789203 & -1.173192 & -3.544846 \\
$\mathrm{H}$ & -3.413856 & -0.463349 & -3.560144 \\
$\mathrm{H}$ & -2.037177 & 0.503167 & -2.968703 \\
$\mathrm{H}$ & -2.820398 & 4.111917 & -1.267847 \\
$\mathrm{H}$ & -1.525167 & 4.053380 & -0.052287 \\
$\mathrm{H}$ & -2.323602 & -0.794105 & 4.328176
\end{tabular}




$\begin{array}{rrrr}\mathrm{H} & -3.730462 & -1.753482 & 3.824467 \\ \mathrm{H} & 3.111637 & -4.113545 & -0.247537 \\ \mathrm{H} & 4.272523 & -2.435650 & -1.574983 \\ \mathrm{H} & 3.859990 & -2.063713 & 0.779930 \\ \mathrm{H} & 1.450421 & -2.515222 & 1.579342 \\ \mathrm{H} & -0.363505 & -2.164684 & 1.521143 \\ \mathrm{H} & 0.579892 & 0.236999 & 2.523898 \\ \mathrm{H} & 4.537732 & 2.820551 & -0.984627 \\ \mathrm{H} & 2.268651 & 3.061384 & -1.961246 \\ \mathrm{H} & 0.296972 & 2.117786 & -0.780643 \\ \mathrm{H} & 2.879945 & 0.671126 & 2.348660 \\ \mathrm{H} & 4.849720 & 1.627522 & 1.172851 \\ \mathrm{H} & -3.165106 & 3.485214 & 0.359219 \\ \mathrm{H} & -3.774887 & 0.016890 & 3.680828\end{array}$

Energies at $0 \mathrm{~K}$ and also H and G @ $298.15 \mathrm{~K}$ : E(0K), E+ZPE(0K), Enthalpy, Free Energy $-1232.949869,-1232.550531,-1232.520485$, $-1232.615306$

\section{hDA 2-MeOH catalyzed (bifurcated mode)}

$\begin{array}{rrrr}\mathrm{C} & -1.872131 & -1.277398 & -0.608941 \\ \mathrm{C} & -0.602298 & -1.562943 & -1.084671 \\ \mathrm{C} & 0.505888 & -1.737099 & -0.232058 \\ \mathrm{H} & -2.069444 & -1.319461 & 0.454930 \\ \mathrm{C} & 0.389602 & 0.518218 & 1.393420 \\ \mathrm{O} & -0.782698 & 0.872096 & 1.018938 \\ \mathrm{C} & -1.921304 & 3.396503 & -1.033554 \\ \mathrm{C} & -2.972977 & -0.052268 & 3.720173 \\ \mathrm{O} & 1.667861 & -1.958556 & -0.907296 \\ \mathrm{~N} & -2.945220 & -0.969748 & -1.345695 \\ \mathrm{Si} & 3.089639 & -2.674739 & -0.368178 \\ \mathrm{O} & -1.892394 & 1.997103 & -1.245450 \\ \mathrm{H} & -1.463565 & 1.586806 & -0.458149 \\ \mathrm{O} & -2.641749 & -0.486618 & 2.415874 \\ \mathrm{H} & -2.013822 & 0.159860 & 2.000566 \\ \mathrm{C} & -4.147907 & -0.449989 & -0.696480 \\ \mathrm{C} & -2.839848 & -0.739863 & -2.782903 \\ \mathrm{H} & -0.390868 & -1.582673 & -2.147313 \\ \mathrm{H} & -5.036454 & -0.962313 & -1.082923 \\ \mathrm{H} & -4.072659 & -0.598001 & 0.382703 \\ \mathrm{H} & -4.227561 & 0.624869 & -0.896821 \\ \mathrm{H} & -2.275281 & -1.546721 & -3.257744 \\ \mathrm{H} & -3.845324 & -0.728459 & -3.210561 \\ \mathrm{H} & -2.352780 & 0.223750 & -2.972702 \\ \mathrm{H} & -2.386782 & 3.857723 & -1.911022 \\ \mathrm{H} & -0.913299 & 3.823744 & -0.917493 \\ \mathrm{H} & -2.091126 & 0.025594 & 4.375686 \\ \mathrm{H} & -3.652492 & -0.791678 & 4.156764 \\ \mathrm{H} & 2.798683 & -4.029364 & 0.172571 \\ \mathrm{H} & 3.932710 & -2.772413 & -1.580324 \\ \mathrm{H} & 3.785906 & -1.878404 & 0.670621 \\ \mathrm{C} & 0.525302 & -1.517153 & 1.158081 \\ \mathrm{H} & 1.426846 & -1.799073 & 1.697434 \\ \mathrm{H} & -0.391318 & -1.692983 & 1.711582 \\ \mathrm{H} & 0.552065 & 0.370190 & 2.475074 \\ \mathrm{C} & 3.894999 & 2.099951 & -0.545263 \\ \mathrm{C} & 2.669450 & 2.092056 & -1.215911 \\ \mathrm{C} & 1.528868 & 1.578048 & -0.598773 \\ \mathrm{C} & 1.605359 & 1.056235 & 0.700780 \\ \mathrm{C} & 2.837719 & 1.071842 & 1.368686\end{array}$

$\begin{array}{rrrr}\mathrm{C} & 3.976096 & 1.590713 & 0.753440 \\ \mathrm{H} & 4.779824 & 2.506972 & -1.027837 \\ \mathrm{H} & 2.599893 & 2.494120 & -2.223533 \\ \mathrm{H} & 0.573523 & 1.588756 & -1.113526 \\ \mathrm{H} & 2.900359 & 0.679481 & 2.382316 \\ \mathrm{H} & 4.923224 & 1.603134 & 1.286858 \\ \mathrm{H} & -2.512774 & 3.674982 & -0.147311 \\ \mathrm{H} & -3.484946 & 0.922837 & 3.721811\end{array}$

Energies at 0K and also H and G @ 298.15 K: $\mathrm{E}(0 \mathrm{~K}), \mathrm{E}+\mathrm{ZPE}(0 \mathrm{~K})$, Enthalpy, Free Energy $-1232.941922,-1232.540664,-1232.512625$, $-1232.600754$

\begin{tabular}{rrrr}
\multicolumn{4}{c}{ hDA 1,4-butanediol catalyzed } \\
starting complex \\
C & -0.887010 & 2.611404 & 0.821451 \\
C & -1.239011 & 1.588517 & 1.635766 \\
C & -0.282413 & 0.724732 & 2.324488 \\
H & 0.165391 & 2.810305 & 0.634354 \\
C & 1.164712 & -0.132719 & -0.963488 \\
O & 0.484612 & 0.754369 & -1.476408 \\
C & -2.078079 & -3.310022 & -0.385754 \\
C & -2.394376 & -0.672959 & -2.476502 \\
$\mathrm{O}$ & -0.881810 & -0.470264 & 2.662005 \\
N & -1.731409 & 3.485789 & 0.173330 \\
Si & -0.153308 & -1.872352 & 3.205134 \\
$\mathrm{O}$ & -1.188886 & -2.325210 & 0.118435 \\
$\mathrm{H}$ & -1.594688 & -1.452415 & -0.083383 \\
$\mathrm{O}$ & -2.169286 & -0.118236 & -1.176810 \\
$\mathrm{H}$ & -1.331588 & 0.393767 & -1.214913 \\
$\mathrm{C}$ & -1.246703 & 4.094270 & -1.057177 \\
$\mathrm{C}$ & -3.160608 & 3.222585 & 0.223925 \\
$\mathrm{H}$ & -2.278111 & 1.306015 & 1.762065 \\
$\mathrm{H}$ & -1.802705 & 5.016864 & -1.256097 \\
$\mathrm{H}$ & -0.188405 & 4.348176 & -0.947175 \\
$\mathrm{H}$ & -1.348895 & 3.429154 & -1.930366 \\
$\mathrm{H}$ & -3.485915 & 3.151202 & 1.266725 \\
$\mathrm{H}$ & -3.695408 & 4.052175 & -0.247192 \\
$\mathrm{H}$ & -3.423401 & 2.283575 & -0.288760 \\
$\mathrm{H}$ & -3.043083 & -3.274116 & 0.148139 \\
$\mathrm{H}$ & -1.620077 & -4.278916 & -0.156134 \\
$\mathrm{H}$ & -1.437872 & -0.813365 & -3.000293 \\
$\mathrm{H}$ & -2.992875 & 0.034028 & -3.070718 \\
$\mathrm{H}$ & 0.417582 & -1.668026 & 4.568934 \\
$\mathrm{H}$ & -1.222405 & -2.891055 & 3.287444 \\
$\mathrm{H}$ & 5.311911 & 1.777481 & -1.851594 \\
$\mathrm{H}$ & 0.956799 & -2.318563 & 2.323349 \\
$\mathrm{H}$ & 0.679713 & -1.026614 & -0.532276 \\
$\mathrm{C}$ & 1.008285 & 0.997842 & 2.604191 \\
$\mathrm{H}$ & 1.666684 & 0.277853 & 3.078608 \\
$\mathrm{H}$ & 1.424982 & 1.973940 & 2.387477 \\
$\mathrm{C}$ & 5.422411 & -0.100200 & -0.792061 \\
$\mathrm{C}$ & 4.707911 & -1.159293 & -0.227247 \\
$\mathrm{C}$ & 3.315512 & -1.161999 & -0.284456 \\
$\mathrm{C}$ & 2.635612 & -0.104213 & -0.905279 \\
$\mathrm{C}$ & 3.359311 & 0.957480 & -1.471692 \\
$\mathrm{C}$ & 4.748311 & 0.957986 & -1.414183 \\
$\mathrm{H}$ & 6.508311 & -0.096895 & -0.748453 \\
$\mathrm{H}$ & 2.745711 & -1.976783 & 0.255971 \\
\hline
\end{tabular}




$$
\begin{array}{llll}
\mathrm{C} & -2.330370 & -3.221249 & -1.895457 \\
\mathrm{C} & -3.139171 & -2.004961 & -2.390884 \\
\mathrm{H} & -1.363166 & -3.258254 & -2.416950 \\
\mathrm{H} & -2.874364 & -4.131856 & -2.185245 \\
\mathrm{H} & -4.031976 & -1.866453 & -1.764592 \\
\mathrm{H} & -3.506464 & -2.225280 & -3.402782
\end{array}
$$

\begin{tabular}{|c|c|c|c|}
\hline \multirow{2}{*}{\multicolumn{4}{|c|}{$\begin{array}{c}\text { TS8 - hDA 1,4-butanediol catalyzed } \\
\text { (cooperative mode) } \\
\text { TS }\end{array}$}} \\
\hline & & & \\
\hline $\mathrm{C}$ & 0.032281 & 2336886 & 021035 \\
\hline $\mathrm{C}$ & & & \\
\hline $\mathrm{C}$ & -1.176595 & & \\
\hline $\mathrm{H}$ & & & \\
\hline $\mathrm{C}$ & -0.34 & 1 & \\
\hline $\mathrm{O}$ & & & \\
\hline $\mathrm{C}$ & & & \\
\hline $\mathrm{C}$ & 3.7 & -1.0 & \\
\hline $\mathrm{O}$ & & & \\
\hline $\mathrm{N}$ & & & \\
\hline $\mathrm{Si}$ & & & \\
\hline $\mathrm{O}$ & & & \\
\hline $\mathrm{H}$ & & & \\
\hline $\mathrm{O}$ & & -0.0 & \\
\hline & & & \\
\hline $\mathrm{C}$ & & & \\
\hline $\mathrm{C}$ & & & \\
\hline $\mathrm{H}$ & & & \\
\hline & & & \\
\hline & & & \\
\hline $\mathrm{H}$ & & & \\
\hline-3 & & & \\
\hline $\mathrm{H}$ & & & \\
\hline & & & \\
\hline & & & \\
\hline $\mathrm{C}$ & & & \\
\hline $\mathrm{H}$ & & & \\
\hline & & & \\
\hline & -2 & & \\
\hline & & & \\
\hline $\mathrm{H}$ & -3 & & \\
\hline$a_{1}$ & -0.5 & -0.2 & \\
\hline & & & \\
\hline & & & \\
\hline $\mathrm{H}$ & & & \\
\hline C & -4.3 & -1.5 & 523 \\
\hline & -3.65 & -0.4 & \\
\hline C & -2.3 & -0.2 & \\
\hline $\mathrm{C}$ & & & \\
\hline C & -2.4 & -2.3 & \\
\hline $\mathrm{C}$ & -3.7 & -2.5 & 875 \\
\hline & -5.408714 & -1.7 & 9261 \\
\hline Н & & & \\
\hline $\mathrm{H}$ & & & \\
\hline $\mathrm{H}$ & -1.991249 & -3.04 & \\
\hline $\mathrm{H}$ & -4.308535 & -3.424690 & -0.787779 \\
\hline$T$ & 2.220364 & -2.496832 & 0.669014 \\
\hline
\end{tabular}

Energies at $0 \mathrm{~K}$ and also H and G @ $298.15 \mathrm{~K}$ : $\mathrm{E}(0 \mathrm{~K}), \mathrm{E}+\mathrm{ZPE}(0 \mathrm{~K})$, Enthalpy, Free Energy -1310.381372, -1309.943736, -1309.914159, $-1310.008719$

$$
\begin{array}{lrrr}
\mathrm{C} & 4.831469 & -1.566016 & -0.410309 \\
\mathrm{H} & 5.188829 & -2.758878 & 1.347003 \\
\mathrm{H} & 4.263594 & -3.600650 & 0.106206 \\
\mathrm{H} & 5.656246 & -1.980924 & -1.004911 \\
\mathrm{H} & 5.243002 & -0.697283 & 0.119553
\end{array}
$$

Energies at $0 \mathrm{~K}$ and also $\mathrm{H}$ and $\mathrm{G} @ 298.15 \mathrm{~K}$ : $\mathrm{E}(0 \mathrm{~K}), \mathrm{E}+\mathrm{ZPE}(0 \mathrm{~K})$, Enthalpy, Free Energy $-1310.362778,-1309.924004,-1309.896201$, $-1309.985004$

\begin{tabular}{rrrr}
\multicolumn{4}{c}{ hDA 1,4-butanediol catalyzed } \\
(bifurcated mode) \\
\multicolumn{4}{c}{ ground state complex } \\
C & -0.860951 & -2.913724 & 0.075359 \\
$\mathrm{C}$ & -1.712764 & -1.927596 & 0.465708 \\
$\mathrm{C}$ & -2.902305 & -1.551555 & -0.291041 \\
$\mathrm{H}$ & -1.067148 & -3.448180 & -0.849464 \\
$\mathrm{C}$ & 0.106654 & 0.861920 & -0.959364 \\
$\mathrm{O}$ & 1.326545 & 0.710236 & -0.899998 \\
$\mathrm{C}$ & 4.981923 & 0.134569 & -1.049112 \\
$\mathrm{C}$ & 5.333571 & 0.858981 & 0.261171 \\
$\mathrm{H}$ & 4.286024 & 0.754775 & -1.631198 \\
$\mathrm{C}$ & 4.415483 & -1.282113 & -0.910937 \\
$\mathrm{C}$ & 4.138055 & 1.382045 & 1.070345 \\
$\mathrm{H}$ & 5.930190 & 0.193507 & 0.903373 \\
$\mathrm{O}$ & -3.862916 & -1.011769 & 0.544901 \\
$\mathrm{~N}$ & 0.239446 & -3.377505 & 0.735565 \\
$\mathrm{Si}$ & -5.368333 & -0.421880 & 0.129725 \\
$\mathrm{O}$ & 3.168546 & -1.369633 & -0.225233 \\
$\mathrm{H}$ & 2.522859 & -0.796034 & -0.679694 \\
$\mathrm{O}$ & 3.365085 & 2.329777 & 0.335758 \\
$\mathrm{H}$ & 2.729633 & 1.827805 & -0.205876 \\
$\mathrm{C}$ & 1.223883 & -4.183649 & 0.023608 \\
$\mathrm{C}$ & 0.708927 & -2.699696 & 1.932208 \\
$\mathrm{H}$ & -1.579187 & -1.407448 & 1.408376 \\
$\mathrm{H}$ & 1.630153 & -4.950521 & 0.693793 \\
$\mathrm{H}$ & 0.741887 & -4.688549 & -0.818424 \\
$\mathrm{H}$ & 2.051569 & -3.564548 & -0.346950 \\
$\mathrm{C}$ & -0.728988 & 4.117845 & 0.755136 \\
$\mathrm{C}$ & 0.013503 & 3.037227 & 0.291716 \\
$\mathrm{H}$ & -2.494007 & 1.296831 & -1.247490 \\
$\mathrm{H}$ & -0.125583 & -2.537522 & 2.621454 \\
$\mathrm{H}$ & 1.447671 & -3.332385 & 2.431364 \\
$\mathrm{H}$ & 1.182449 & -1.735015 & 1.701532 \\
$\mathrm{H}$ & 5.890116 & 0.054312 & -1.663585 \\
$\mathrm{H}$ & 5.971501 & 1.719298 & 0.019940 \\
$\mathrm{H}$ & 4.335595 & -1.734129 & -1.914104 \\
$\mathrm{H}$ & 5.109872 & -1.904909 & -0.333845 \\
$\mathrm{H}$ & 3.514026 & 0.546356 & 1.409670 \\
$\mathrm{H}$ & 4.509868 & 1.907749 & 1.958044 \\
$\mathrm{H}$ & -6.241796 & -1.481182 & -0.442919 \\
$\mathrm{H}$ & -5.948595 & 0.084161 & 1.394647 \\
$\mathrm{H}$ & -5.273605 & 0.689057 & -0.858648 \\
$\mathrm{C}$ & -3.089931 & -1.663597 & -1.623846 \\
$\mathrm{H}$ & -4.039926 & -1.447064 & -2.101820 \\
$\mathrm{H}$ & -2.297044 & -2.035550 & -2.262033 \\
$\mathrm{H}$ & -2.008173 & 2.097388 & -0.694773 \\
$\mathrm{H}$ & & -0.430661
\end{tabular}




$$
\begin{array}{rrrr}
\mathrm{H} & -2.674004 & 5.044307 & 0.856773 \\
\mathrm{H} & -0.240592 & 4.903534 & 1.324409 \\
\mathrm{H} & 1.076944 & 2.966414 & 0.502717 \\
\mathrm{H} & -0.515154 & 0.083149 & -1.433616
\end{array}
$$

Energies at $0 \mathrm{~K}$ and also H and G @ 298.15 K: $\mathrm{E}(0 \mathrm{~K}), \mathrm{E}+\mathrm{ZPE}(0 \mathrm{~K})$, Enthalpy, Free Energy $-1310.377908,-1309.940355,-1309.910387$, $-1310.005849$

\section{hDA 1,4-butanediol catalyzed (bifurcated mode)}

\begin{tabular}{lrrr} 
& \multicolumn{3}{c}{ TS } \\
C & -0.954496 & 2.473201 & -0.548239 \\
C & 0.237810 & 2.470190 & 0.154300 \\
C & 1.432164 & 1.944600 & -0.373764 \\
H & -0.969689 & 2.174846 & -1.588723 \\
C & 0.606961 & -0.459464 & -1.396329 \\
O & -0.620048 & -0.217980 & -1.100358 \\
C & -3.895471 & -1.309955 & 0.140858 \\
$\mathrm{C}$ & -3.542539 & -2.804884 & 0.185467 \\
$\mathrm{H}$ & -3.562863 & -0.896358 & -0.821390 \\
$\mathrm{C}$ & -3.361675 & -0.443169 & 1.286500 \\
$\mathrm{C}$ & -2.108573 & -3.145627 & -0.247711 \\
$\mathrm{H}$ & -3.718030 & -3.199396 & 1.197914 \\
$\mathrm{O}$ & 2.460850 & 1.960443 & 0.507412 \\
$\mathrm{~N}$ & -2.144132 & 2.840572 & -0.056201 \\
$\mathrm{Si}$ & 4.112307 & 1.735180 & 0.250838 \\
$\mathrm{O}$ & -1.952120 & -0.308099 & 1.370856 \\
$\mathrm{H}$ & -1.553593 & -0.224527 & 0.473689 \\
$\mathrm{O}$ & -1.836812 & -2.724636 & -1.576499 \\
$\mathrm{H}$ & -1.497710 & -1.808376 & -1.523365 \\
$\mathrm{C}$ & -3.332067 & 2.880861 & -0.898694 \\
$\mathrm{C}$ & -2.308819 & 3.100605 & 1.372581 \\
$\mathrm{H}$ & 0.277586 & 2.813552 & 1.181147
\end{tabular}

$\begin{array}{rrrr}\mathrm{H} & -3.767010 & 3.888019 & -0.904767 \\ \mathrm{H} & -3.065270 & 2.608884 & -1.921655 \\ \mathrm{H} & -4.087492 & 2.174299 & -0.536333 \\ \mathrm{H} & -1.720998 & 3.972445 & 1.683650 \\ \mathrm{H} & -3.361466 & 3.303120 & 1.576656 \\ \mathrm{H} & -1.993858 & 2.217369 & 1.939057 \\ \mathrm{H} & -4.990096 & -1.202266 & 0.153104 \\ \mathrm{H} & -4.227128 & -3.339774 & -0.486066 \\ \mathrm{H} & -3.840429 & 0.552189 & 1.212674 \\ \mathrm{H} & -3.684356 & -0.866651 & 2.247368 \\ \mathrm{H} & -1.388311 & -2.715599 & 0.459693 \\ \mathrm{H} & -1.982048 & -4.235994 & -0.224202 \\ \mathrm{H} & 4.590904 & 2.700655 & -0.774690 \\ \mathrm{H} & 4.722393 & 2.035668 & 1.564461 \\ \mathrm{H} & 4.441674 & 0.357482 & -0.176159 \\ \mathrm{C} & 1.547429 & 1.282947 & -1.615677 \\ \mathrm{H} & 2.549195 & 1.029112 & -1.955407 \\ \mathrm{H} & 0.907851 & 1.640534 & -2.416457 \\ \mathrm{H} & 0.821438 & -0.714466 & -2.451008 \\ \mathrm{C} & 3.212044 & -2.700672 & 1.215419 \\ \mathrm{C} & 2.138620 & -1.986044 & 1.752954 \\ \mathrm{C} & 1.286125 & -1.256230 & 0.922611 \\ \mathrm{C} & 1.507603 & -1.225839 & -0.461510 \\ \mathrm{C} & 2.582433 & -1.951949 & -0.992967 \\ \mathrm{C} & 3.429399 & -2.686634 & -0.164384 \\ \mathrm{H} & 3.867611 & -3.274125 & 1.865761 \\ \mathrm{H} & 1.955738 & -2.005227 & 2.824392 \\ \mathrm{H} & 0.437573 & -0.729291 & 1.345845 \\ \mathrm{H} & 2.746516 & -1.949024 & -2.069323 \\ \mathrm{H} & 4.251853 & -3.253082 & -0.593977\end{array}$

Energies at $0 \mathrm{~K}$ and also $\mathrm{H}$ and $\mathrm{G} @ 298.15 \mathrm{~K}$ : $\mathrm{E}(0 \mathrm{~K}), \mathrm{E}+\mathrm{ZPE}(0 \mathrm{~K})$, Enthalpy, Free Energy $-1310.356735,-1309.917916,-1309.890054$, $-1309.977242$ 
C. MP2 Energies (MP2/6-31G(d)//B3LYP/6-31G(d))

2-MeOH ground state

MP2 electronic energy $\quad E_{\text {rel }}$

comples (cooperative

mode)

2-MeOH Step 1 TS

(cooperative mode)

2-MeOH Intermediate

(cooperative mode)

2-MeOH Step 2 TS

(cooperative mode)

2-MeOH product complex

(cooperative mode)

$-822.733441$

0

$-822.699577$

21.2

$\begin{array}{ll}-822.716923 & 10.4\end{array}$

$-822.717693$

9.9

$-822.763188$

$-18.7$ 
D. Charge Separation between diene component and dieophile-catalyst complex and charge on carbonyl oxygen (CHELPG B3LYP/6-31G(d))

$\begin{array}{ccc}\text { Hetero-Diels Alder Reaction } & \text { charge separation } & \text { carbonyl oxygen } \\ \text { uncatalyzed TS } & 0.35 & -0.61 \\ \text { 1-MeOH catalyzed TS } & 0.32 & -0.62 \\ \text { 2-MeOH TS (cooperative mode) } & 0.25 & -0.69 \\ \text { 2-MeOH TS (bifurcated mode) } & 0.20 & -0.64 \\ \text { 1.4-butanediol catalyzed TS (cooperative } & 0.31 & -0.70 \\ \text { mode) } & & \\ \text { 1.4-butanediol catalyzed TS (bifurcated } & 0.30 & -0.62 \\ \text { mode) } & & \\ \text { Diels-Alder Reaction } & 0.32 & -0.58 \\ \text { uncatalyzed TS } & 0.32 & -0.62 \\ \text { 1-MeOH catalyzed } & 0.24 & -0.57 \\ \text { 2-MeOH TS (bifurcated mode) } & 0.50 & -0.57 \\ \text { 2-MeOH Intermediate (bifurcated mode) } & 0.25 & -0.61 \\ \text { 2-MeOH TS (cooperative mode) } & 0.44 & -0.61 \\ \text { 2-MeOH Intermediate (cooperative mode) } & 0.30 & -0.62 \\ \text { 1,4-butanediol TS } & 0.53 & -0.62 \\ \text { 1,4-butanediol Intermediate } & & -0.44 \\ \text { Reference Charges } & & -0.48 \\ \text { s-trans-acrolein: carbonyl oxygen charge } & & \\ \text { s-trans-acrolein-butanediol (cooperative } & & -0.42 \\ \text { mode) complex: carbonyl oxygen charge } & & -0.43 \\ \text { benzaldehyde: carbonyl oxygen charge } & & \\ \text { benzaldehyde-butanediol (cooperative } & & \\ \text { mode) complex: carbonyl oxygen charge } & & \end{array}$

Atomic charges have been calculated according to the CHELPG scheme using the DFT KohnSham orbitals and are given in fractions of the elementary charge unit e. ${ }^{1}$ Charge separation was calculated from the sum of the atomic charges of the diene component. The charge on the carbonyl oxygen is the CHELPG charge for the single oxygen atom.

All of the cycloaddition reactions of Rawal-type dienes evaluated in this study proceed via highly polarized transition states. These transition states are highly asynchronous and are zwitterionic in nature, as evidenced by the charge separation between the reactants at the TS and by the development of either enolate or alkoxide character at the carbonyl oxygen at the TS.

\footnotetext{
${ }^{1}$ Breneman, D. M.; Wiberg, K. B. J. Comp. Chem. 1990, 11, 361-373.
} 


\section{E. Hydrogen Bond Strength Increases as Enolate Character Develops.}<smiles></smiles><smiles>CC=C[O-]</smiles><smiles>C[12CH2]</smiles>

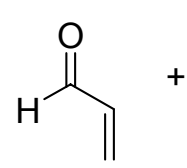<smiles></smiles>

$\Delta \mathrm{H}_{298}=-23.7 \mathrm{kcal} / \mathrm{mol}$ at B3LYP/6-31G(d)

The maximum catalytic effect of a hydrogen bond catalyst was determined from the above isodesmic reaction. Hydrogen bond strength with 1,4-butanediol (cooperative mode) was found to be 23.7 $\mathrm{kcal} / \mathrm{mol}$ stronger in the gas phase with E-1-propen-1-ol, as opposed to acrolein. Although this simple model does not consider solvation and therefore overestimates the strength of the enolate-diol hydrogen bond interaction, it is in qualitative agreement with the earlier computational studies of hydrogen bond strength of Remer and Jensen. ${ }^{2}$

Hydrgogen bond lengths for ground state complexes, TS complexes and intermediates are presented in the table below. As the anionic character of the hydrogen bond acceptor increases the hydrogen bond length becomes shorter.

Table of Hydrogen Bond Distances.

\section{Hydrogen bond distances $(\mathrm{O}-\mathrm{H}-\mathrm{-}-\mathrm{O}=\mathrm{C})$}

\begin{tabular}{|c|c|c|c|c|}
\hline & & $\begin{array}{l}\text { Ground } \\
\text { State } \\
\text { Complex }\end{array}$ & TS & Intermediate \\
\hline \multirow[t]{4}{*}{ DA reaction } & 1-MeOH & $1.85 \AA$ & $1.74 \AA$ & $\mathrm{n} / \mathrm{a}$ \\
\hline & 2-MeOH (cooperative mode) & $1.78 \AA$ & $1.68 \AA$ & $1.63 \AA$ \\
\hline & 2-MeOH (bifurcated mode) ${ }^{*}$ & $1.89 \AA$ & $1.80 \AA$ & $1.72 \AA$ \\
\hline & 1,4-butanediol (cooperative mode) & $1.80 \AA$ & $1.69 \AA$ & $1.60 \AA$ \\
\hline \multirow{5}{*}{$\begin{array}{l}\text { hDA } \\
\text { reaction }\end{array}$} & 1-MeOH & $1.88 \AA$ & $1.72 \AA$ & $\mathrm{n} / \mathrm{a}$ \\
\hline & 2-MeOH (cooperative mode) & $1.81 \AA$ & $1.61 \AA$ & $\mathrm{n} / \mathrm{a}$ \\
\hline & 2-MeOH (bifurcated mode) ${ }^{*}$ & $1.89 \AA$ & $1.75 \AA$ & $\mathrm{n} / \mathrm{a}$ \\
\hline & 1,4-butanediol (cooperative mode) & $1.87 \AA$ & $1.60 \AA$ & $\mathrm{n} / \mathrm{a}$ \\
\hline & 1,4-butanediol (bifurcated mode) ${ }^{*}$ & $1.93 \AA$ & $1.85 \AA$ & $\mathrm{n} / \mathrm{a}$ \\
\hline
\end{tabular}

\footnotetext{
${ }^{2}$ Remer, L. C.; Jensen, J. H. J. Phys. Chem. A 2000, 104, 9266-9275
} 